\title{
TODA BRACKETS AND CUP-ONE SQUARES FOR RING SPECTRA
}

\author{
HANS-JOACHIM BAUES AND FERNANDO MURO
}

\begin{abstract}
In this paper we prove the laws of Toda brackets on the homotopy groups of a connective ring spectrum and the laws of the cup-one square in the homotopy groups of a commutative connective ring spectrum.
\end{abstract}

Contents

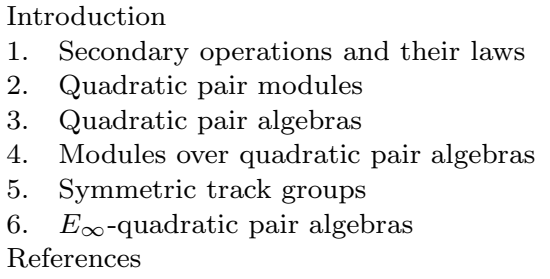

INTRODUCTION

Secondary homotopy operations such as triple Toda brackets are defined on the homotopy groups of a ring spectrum $R$ enriching the ring structure of $\pi_{*} R$. Toda established in Tod62 a set of relations for Toda brackets in the stable homotopy groups of spheres, Alexander claimed these relations for some cobordism rings in Ale72, and we show here that the Toda relations are, in fact, satisfied for any connective ring spectrum $R$ (Definition 1.2 and Theorem 1.3). Moreover, if the ring spectrum $R$ is commutative further relations proved by Toda for the sphere spectrum, such as the Jacobi identity, are shown to be satisfied in general (Definition 1.8 and Theorem [1.9).

If $R$ is commutative a new secondary homotopy operation appears, namely the cup-one square. This operation was studied in BMMS86 in the context of $H_{\infty}$-ring spectra. The operation in BMMS86] is, however, only defined up to an indeterminacy. We show that one can extract from this undetermined operation a fully determined cup-one square and we compute its behaviour with respect to sums and products in $\pi_{*} R$, as well as its relation to Toda brackets (Definition 1.8 and Theorem 1.9). This is done by carrying out a careful analysis in the "symmetric track groups" introduced in BM06c. In this way we are able to compute explicitly the deviation of the cup-one square from additivity and from being a quadratic derivation, which was only computed in BMMS86 up to an unknown constant, see (T11) and (T12) in Definition 1.8.

Date: February 2, 2008.

1991 Mathematics Subject Classification. 18G50,55P42,55Q35.

Key words and phrases. Ring spectrum, Toda bracket, cup-one square, quadratic pair algebra. The second author was partially supported by the project MTM2004-01865 and the MEC postdoctoral fellowship EX2004-0616. 
For the proofs we use the algebraic framework of $\left(E_{\infty^{-}}\right)$quadratic pair algebras, which are algebraic models of (commutative) ring spectra extending the homotopy groups and codifying all secondary operations, see [BM06a].

The statements of the homotopical results are in the first section, which can be regarded as a continuation of this introduction. The rest of sections are purely algebraic and contain all proofs.

\section{SECONDARY OPERATIONS AND THEIR LAWS}

A (commutative) ring spectrum is a (commutative) monoid in the closed symmetric monoidal model category of symmetric spectra of compactly generated topological spaces defined in MMSS01, 12]. The monoidal structure is given by the smash product $X \wedge Y$ and the unit object is the sphere spectrum $S$. A symmetric spectrum is connective if its homotopy groups vanish in negative dimensions.

The homotopy groups of a connetive ring spectrum $\pi_{*} R$ form an $\mathbb{N}$-graded ring, where $\mathbb{N}=\{0,1,2, \ldots\}$. All rings and modules in this paper will be $\mathbb{N}$-graded and the degree of a homogeneous element $x$ will be denoted by $|x|$. Ungraded objects are regarded as graded objects concentrated in degree 0 . The degree of a homogeneous element $a \in \pi_{*} R$ is denoted by $|a|$. The ring $\pi_{*} R$ is equipped with secondary homotopy operations called Toda brackets. The Toda bracket of three homogeneous elements

is a coset of

$$
\langle a, b, c\rangle \subset \pi_{|a|+|b|+|c|+1} R
$$

$$
\left(\pi_{|a|+|b|+1} R\right) \cdot c+a \cdot\left(\pi_{|b|+|c|+1} R\right)
$$

which is defined whenever $a b=0$ and $b c=0$. This operation was first considered by Toda for the sphere spectrum $S$, see Tod62. In Ale72] one finds a construction of Toda brackets for various cobordism spectra under the name of Massey products. We consider in [BM06a] two equivalent definitions of Toda brackets on the homotopy groups of a ring spectrum $R$. Both definitions use the model category of right $R$-modules, see [MMSS01, 12]. One of the definitions uses Toda brackets for triangulated categories in the sense of [Hel68] applied to the homotopy category of $R$-modules. This is also the definition adopted in Sag06. The alternative definition uses tracks, i.e. homotopy classes of homotopies, in the model category of $R$-modules. We now recall this definition.

The homotopy group $\pi_{n} R$ coincides with the group of morphisms from the $n$ fold suspension $\Sigma^{n} R \rightarrow R$ in the homotopy category of right $R$-modules. We can suppose without loss of generality that $R$ is a fibrant ring spectrum. In that case the elements $a, b, c \in \pi_{*} R$ can be realized by maps $\bar{a}, \bar{b}, \bar{c}$ in the category of $R$-modules. The vanishing hypothesis $a b=0$ and $b c=0$ imply the existence of null-homotopies

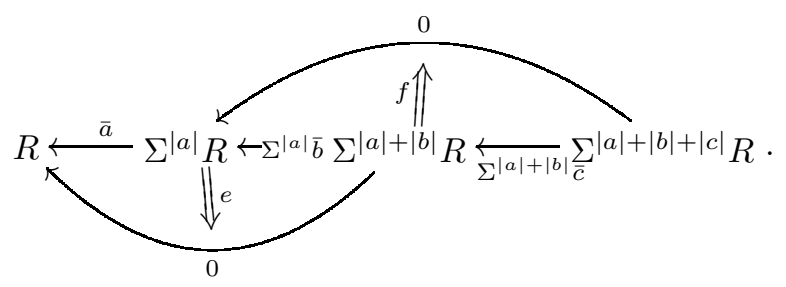

The pasting of this diagram is a self-track of the trivial map $0: \Sigma^{|a|+|b|+|c|} R \rightarrow R$. Such a self-track is the same as a homotopy class

$$
\Sigma^{|a|+|b|+|c|+1} R \longrightarrow R,
$$

which by definition is a generic element of the Toda bracket $\langle a, b, c\rangle$.

The next definition encodes the secondary algebraic structure of the homotopy ring $\pi_{*} R$ endowed with the Toda brackets. 
Definition 1.2. Let $A$ be a ring and let $M$ be an $A$-bimodule. We say that $A$ has secondary oprations with coefficients in $M$ if there is given a bimodule homomorphism

$$
\cdot \eta: A \otimes \mathbb{Z} / 2 \longrightarrow M
$$

and for any three homogeneous elements $a, b, c \in A$ with $a b=0$ and $b c=0$ there is defined a coset (the bracket operation)

$$
\langle a, b, c\rangle \subset M_{|a|+|b|+|c|} \text { of } M_{|a|+|b|} \cdot c+a \cdot M_{|b|+|c|}
$$

satisfying the following relations (whenever the brackets are defined):

(T1) $0 \in\langle a, b, c\rangle$ provided $a, b$ or $c$ is zero.

(T2) $\langle a, b, c\rangle$ is linear in each variable, i.e.

$$
\begin{aligned}
& \left\langle a+a^{\prime}, b, c\right\rangle \subset\langle a, b, c\rangle+\left\langle a^{\prime}, b, c\right\rangle, \\
& \left\langle a, b+b^{\prime}, c\right\rangle=\langle a, b, c\rangle+\left\langle a, b^{\prime}, c\right\rangle, \\
& \left\langle a, b, c+c^{\prime}\right\rangle \subset\langle a, b, c\rangle+\left\langle a, b, c^{\prime}\right\rangle .
\end{aligned}
$$

(T3) $a \cdot\langle b, c, d\rangle \subset\langle a \cdot b, c, d\rangle$ and $\langle a, b, c\rangle \cdot d \subset\langle a, b, c \cdot d\rangle$.

(T4) $\langle a \cdot b, c, d\rangle \subset\langle a, b \cdot c, d\rangle \supset\langle a, b, c \cdot d\rangle$.

(T5) $0 \in\langle a, b, c\rangle \cdot d+a \cdot\langle b, c, d\rangle$,

(T6) $a \cdot \eta \in\langle 2, a, 2\rangle$.

The desuspension $\Sigma^{-1} A$ of a ring $A$ is the $A$-bimodule with $\left(\Sigma^{-1} A\right)_{n}=A_{1+n}$ and bimodule structure

$$
a \cdot\left(\Sigma^{-1} b\right) \cdot c=(-1)^{|a|} \Sigma^{-1}(a \cdot b \cdot c) .
$$

Here $a, b, c \in A$ are homogeneous elements and given $x \in A_{n}$ with $n \geq 1$ we denote by $\Sigma^{-1} x$ to the corresponding element in $\left(\Sigma^{-1} A\right)_{n-1}$. One can similarly define the desuspension of a right $A$-module, for which there are no signs involved in the action.

Theorem 1.3. Let $R$ be a connective ring spectrum. The ring $\pi_{*} R$ has secondary operations with coefficients in $\Sigma^{-1} \pi_{*} R$, in the sense of Definition 1.2. The homomorphism $\cdot \eta$ is defined by multiplication from the right by the image of the stable Hopf map $0 \neq \eta \in \pi_{1} S \cong \mathbb{Z} / 2$ under the ring homomorphism $\pi_{*} S \rightarrow \pi_{*} R$ induced by the unit $S \rightarrow R$ of the ring spectrum, and the bracket operation is given by Toda brackets.

This theorem follows from Theorems 3.4 and 3.5 below.

Remark 1.4. Alexander considered in [Ale72, Definition 2.1] a notion of a ring with secondary operations similar to Definition 1.2. Our relations (T1)-(T5) correspond to relations (1)-(5) in Ale72, Definition 2.1] if $M=\Sigma^{-1} A$. The homomorphism $\eta$, and therefore (T6) above, are not considered in [Ale72, Definition 2.1], although they appear in particular examples, see Ale72, Theorem 6.2]. Relation (6) in Ale72, Definition 2.1] is not codified by Definition 1.2 since it is not a secondary relation, it has higher order. Alexander's relations are claimed in Ale72 for some cobordism rings. These rings may arise as the homotopy groups of connective spectra, see [Ale72, Section 4], which may be given the structure of ring spectra as in [Sch04, Example 4.15].

Alexander's relations (1)-(6) coincide with the relations (3.5)-(3.8) in Tod62. previously proved by Toda for the sphere spectrum.

The homotopy groups $\pi_{*} M$ of a connective right $R$-module $M$ form a right $\pi_{*} R$-module which is also endowed with Toda brackets

$$
\langle a, b, c\rangle \in \pi_{|a|+|b|+|c|+1} M
$$


defined for homogeneous elements $a \in \pi_{*} M$ and $b, c \in \pi_{*} R$ whenever $a b=0$ and $b c=0$, which is a coset of

$$
\left(\pi_{|a|+|b|+1} M\right) \cdot c+a \cdot\left(\pi_{|b|+|c|+1} R\right)
$$

These Toda brackets are defined replacing $R$ by $M$ on the left hand side of diagram (1.1).

The folowing definition codifies the algebraic structure of Toda brackets in $\pi_{*} M$.

Definition 1.5. Let $A$ be a ring with secondary operations with coefficients in the $A$-bimodule $M$ and let $N$ and $L$ be right $A$-modules. We say that $N$ has secondary operations with coefficients $L$ if a right $A$-module homomorphism

$$
\cdot: N \otimes_{A} M \longrightarrow L
$$

is given and for any three homogeneous elements $a \in N, b, c \in A$ with $a b=0$ and $b c=0$ there is defined a coset (the bracket operation)

$$
\langle a, b, c\rangle \subset L_{|a|+|b|+|c|} \text { of } L_{|a|+|b|} c+a M_{|b|+|c|}
$$

satisfying relations (T1)-(T5) in Definition 1.2 for $a, a^{\prime} \in N$ and $b, b^{\prime}, c, c^{\prime}, d \in A$.

Theorem 1.6. Let $R$ be a connective ring spectrum and let $K$ be a connective right $R$-module. The right $\pi_{*} R$-module $\pi_{*} K$ has secondary operations with coefficients in $\Sigma^{-1} \pi_{*} K$, in the sense of Definition 1.5. The homomorphism

$$
\cdot: \pi_{*} K \otimes_{\pi_{*} R} \Sigma^{-1} \pi_{*} R \longrightarrow \Sigma^{-1} \pi_{*} K
$$

is defined by the right $\pi_{*} R$-module structure of $\pi_{*} K$ according to the formula

$$
m \cdot\left(\Sigma^{-1} a\right)=(-1)^{|m|} \Sigma^{-1}(m \cdot a),
$$

and the bracket operation is given by Toda brackets.

This theorem follows from Theorems 4.3 and 4.4 below.

The homotopy groups of a commutative ring spectrum $\pi_{*} R$ form a commutative ring (in the graded sense) which carries, appart from Toda brackets, an additional operation called cup-one square,

$$
S q_{1}: \pi_{2 n} R \longrightarrow \pi_{4 n+1} R,
$$

defined as follows. Let $L R$ be a fibrant replacement of $R$ in the category of all ring spectra. The ring spectrum $L R$ is no longer commutative, but it remains commutative up to a coherent track $\alpha_{1}$ (i.e. a homotopy class of homotopies) satisfying the idempotence and the hexagon axioms for symmetric monoidal categories, compare BM06a, Lemma 16.2]. Given $a \in \pi_{2 n} R$ we take a representative $\bar{a}: S^{2 n} \rightarrow L R$ where the spectrum $S^{m}$ is the $m$-fold suspension of the sphere spectrum $S, S^{m}=\Sigma^{m} S$. The symmetry isomorphism for the smash square of an even-dimensional sphere $\tau_{\wedge}: S^{2 n} \wedge S^{2 n} \cong S^{2 n} \wedge S^{2 n}$ is homotopic to the identity. We can choose a track $\hat{\tau}_{2 n, 2 n}: \tau_{\wedge} \Rightarrow 1_{S^{2 n} \wedge S^{2 n}}$, there are two such choices. Consider the following diagram where $\mu$ is the product in $L R$.

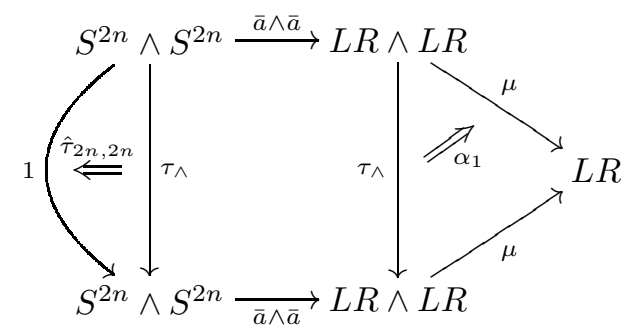


The pasting of this diagram is a self-track of $\mu(\bar{a} \wedge \bar{a})$. The classical Barcus-BarrattRutter isomorphism allows us to identify this self-track with a homotopy class

$$
S q_{1}(a): S^{4 n+1}=\Sigma\left(S^{2 n} \wedge S^{2 n}\right) \longrightarrow R
$$

measuring the difference between the pasting of (1.7) and the identity self-track on $\mu(\bar{a} \wedge \bar{a})$. This element $S q_{1}(a) \in \pi_{4 n+1} R$ is the cup-one square of $a$. One can check that $S q_{1}(a)$ does not depend on the representative $\bar{a}$. However in general it does depend on the choice of $\hat{\tau}_{2 n, 2 n}$. The difference bewteen the two possible definitions of $S q_{1}$, depending on the choice of $\hat{\tau}_{2 n, 2 n}$, is computed in [BM06a, Lemma 9.11], see Lemma 6.2.

The relations between Toda brackets and cup-one squares in the homotopy groups of a commutative ring spectrum is algebraically encoded by the following definition.

Definition 1.8. A commutative ring $A$ with commutative secondary operations with coefficients in an $A$-module $M$ is a ring with secondary operations in the sense of Definition 1.2 together with maps

$$
S q_{1}: A_{2 n} \longrightarrow M_{4 n}, \quad n \geq 0,
$$

such that the following further axioms hold:

(T7) $\langle a, b, c\rangle=(-1)^{|a||b|+|b||c|+|c||a|+1}\langle c, b, a\rangle$.

(T8) $0 \in(-1)^{|a||c|}\langle a, b, c\rangle+(-1)^{|b||a|}\langle b, c, a\rangle+(-1)^{|c||b|}\langle c, a, b\rangle$,

(T9) for $|a|$ odd $\langle a, b, a\rangle \cap(-1)^{|a||b|}\langle b, a, 2 a\rangle \neq \emptyset$,

(T10) for $|a|$ even $(-1)^{|a||b|} b \cdot S q_{1}(a) \in\langle a, b, a\rangle$,

(T11) $S q_{1}(a+b)=S q_{1}(a)+S q_{1}(b)+\left(\frac{|a|}{2}+1\right) \cdot a \cdot b \cdot \eta$,

(T12) $S q_{1}(a \cdot b)=a^{2} \cdot S q_{1}(b)+S q_{1}(a) \cdot b^{2}+\frac{|a||b|}{4} \cdot a^{2} \cdot b^{2} \cdot \eta$.

Theorem 1.9. Let $R$ be a connective commutative ring spectrum. The ring $\pi_{*} R$ has commutative secondary operations with coefficients in $\Sigma^{-1} \pi_{*} R$, in the sense of Definition 1.8. The operation $S q_{1}$ is the cup-one square for an explicit choice of tracks $\hat{\tau}_{2 n, 2 n}, n \geq 0$, and the rest of the structure is given by Theorem 1.3.

This theorem follows from Theorems 6.3 and 6.4 below.

Remark 1.10. There is also a notion of a commutative ring with commutative secondary operations in [Ale72, Definition 2.1]. This notion however does not codify the operation $S q_{1}$. Our relations (T7) and (T8) correspond to relations (7) and (8) in [Ale72, Definition 2.1] if $M=\Sigma^{-1} A$. These relations are claimed in Ale72. for some commutative cobordism rings which may arise as the homotopy groups of commutative connective ring spectra, see Remark 1.4. Notice that there is a misprint in the exponent of $(-1)$ in relation (8) of [Ale72, Definition 2.1]. It does not include the summand +1 . This misprint does not appear in Toda's relations for the case of the sphere spectrum, see (3.9) in Tod62. Relation (T9) corresponds to the first half of Toda's (3.10) in Tod62. The second half is a weak version of (T10) which avoids the use of $S q_{1}$.

$H_{\infty}$-ring spectra in the sense of BMMS86] are an early version of commutative ring spectra "up to homotopy". The operations $S q_{1}$ are closely related to the power operations for $H_{\infty}$-ring spectra considered in BMMS86, V.1]. More precisely, the operation $P^{n+1}$ in [BMMS86, V] on $\pi_{n}$ for $p=2$ and $n=2 k$ corresponds to the set $P^{n+1}(a)=\left\{S q_{1}(a), S q_{1}(a)+a^{2} \cdot \eta\right\}$. Then relation (T11) above implies the deviation from additivity indicated in [BMMS86, $\mathrm{V}$ Table 1.3] and relation (T12) implies the first equation of [BMMS86, Proposition V.1.10] and gives the explicit value for the constant $c_{n, m}$ which is not determined in [BMMS86]. Maybe one of the most surprising implications of Theorem 1.9 is the existence of choices 
$S q_{1}(a) \in P^{n+1}(a)$, for $p=2, n=2 k$, and $k \geq 0$, satisfying relations (T11) and (T12).

Any commutative ring $A$ with commutative secondary operations with coefficients in an $A$-module $M$ has the following remarkable property. If we define $S q_{1}^{\omega}(a)=S q_{1}(a)+a^{2} \cdot \eta$, then $S q_{1}^{\omega}$ also satisifies the axioms in Definition 1.8

In the following proposition we record some additional relations between cup-one squares derived from Definition 1.8.

Proposition 1.11. Let $A$ be a commutative ring with commutative secondary operations with coefficients in the A-module $M$. Then

(1) $S q_{1}(1)=0$,

(2) $S q_{1}(2)=1 \cdot \eta$

(3) $2 \cdot S q_{1}(a)=\frac{|a|}{2} \cdot a^{2} \cdot \eta$,

(4) $S q_{1}(2 \cdot a)=a^{2} \cdot \eta$.

Proof. Equation (1) follows from (T12) applied to $a=b=1$, and (2) follows from (T11) and (1). Applying (T11) to $a+a$ and (T12) to $2 \cdot a$ we obtain the equation

$$
2 \cdot S q_{1}(a)+\left(\frac{|a|}{2}+1\right) \cdot a^{2} \cdot \eta=4 \cdot S q_{1}(a)+a^{2} \cdot \eta \text {. }
$$

Here we use (2) to identify $S q_{1}(2) \cdot a^{2}=a^{2} \cdot \eta$. Equation (3) follows from this one. Finally (4) follows from (T11) and (3).

Similar relations are shown in BMMS86, V.1] for the power operations on the homotopy groups of $H_{\infty}$-ring spectra.

Remark 1.12. By Proposition 1.11(2) the structure homomorphism $\cdot \eta$ of a commutative ring with commutative secondary operations is determined by the operation $S q_{1}$, so one could restate Definition 1.8 just in terms of the bracket and $S q_{1}$.

\section{Quadratic Pair Modules}

The topological theorems of this paper are proved by using the quadratic algebraic models for ring and module spectra defined in [BM06a]. In this section we recall the basics on the necessary quadratic algebra, see BP99, BJP05.

A quadratic pair module $C$ is a diagram

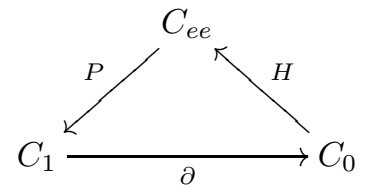

where $C_{0}$ and $C_{1}$ are groups, $C_{e e}$ is an abelian group, $P$ and $\partial$ are homomorphisms, and $H$ is a quadratic map, i.e. the crossed efect

$$
\left(x_{1} \mid x_{2}\right)_{H}=H\left(x_{1}+x_{2}\right)-H\left(x_{2}\right)-H\left(x_{1}\right), \quad x_{i} \in C_{0},
$$

is bilinear. Moreover, the following equations hold for $x, x_{i} \in C_{0}, s_{i} \in C_{1}$ and $a \in C_{e e}$.

$$
\begin{aligned}
P H \partial P(a) & =P(a)+P(a), \\
H(x+\partial P(a)) & =H(x)+H \partial P(a), \\
P H\left(\partial\left(s_{1}\right)+\partial\left(s_{2}\right)\right) & =P H \partial\left(s_{1}\right)+P H \partial\left(s_{2}\right)+\left[s_{1}, s_{2}\right], \\
\partial P H\left(x_{1}+x_{2}\right) & =\partial P H\left(x_{1}\right)+\partial P H\left(x_{2}\right)+\left[x_{1}, x_{2}\right],
\end{aligned}
$$

see [BJP06, 2.4]. Here $[\alpha, \beta]=-\alpha-\beta+\alpha+\beta$ denotes the commutator bracket of two elements $\alpha, \beta \in G$ in a group $G$. 
It follows from the axioms that $C_{0}$ and $C_{1}$ are groups of nilpotency class 2 , so commutators are central and bilinear, $\partial\left(C_{1}\right)$ is a normal subgroup of $C_{0}$, and $P$ and Ker $\partial$ are central. The quadratic map $H$ satisfies

$$
\begin{aligned}
H(0) & =0, \\
H(-x) & =-H(x)+(x \mid x)_{H} .
\end{aligned}
$$

For any quadratic pair module the function

$$
T=H \partial P-1: X_{e e} \longrightarrow X_{e e}
$$

is an involution, i.e. a homomorphism with $T^{2}=1$. Using (M4) cone can check that

$$
T\left(x_{1} \mid x_{2}\right)_{H}=-\left(x_{2} \mid x_{1}\right)_{H} .
$$

By (M1) $T$ satisfies $P T=P$, therefore

$$
P\left(x_{1} \mid x_{2}\right)_{H}=-P\left(x_{2} \mid x_{1}\right)_{H} .
$$

Moreover,

$$
\Delta: C_{0} \longrightarrow C_{e e}: x \mapsto(x \mid x)_{H}-H(x)+T H(x)
$$

is a homomorphism which satisfies $P \Delta(x)=P(x \mid x)_{H}$.

The homology of a quadratic pair module $C$ is given by the abelian groups defined as

$$
\begin{aligned}
h_{0} C & =\operatorname{Coker}\left(\partial: C_{1} \rightarrow C_{0}\right), \\
h_{1} C & =\operatorname{Ker}\left(\partial: C_{1} \rightarrow C_{0}\right) .
\end{aligned}
$$

The $k$-invariant of $C$ is the natural homomorphism

$$
\cdot \eta: h_{0} C \otimes \mathbb{Z} / 2 \longrightarrow h_{1} C
$$

given by the formula

$$
x \cdot \eta=P(x \mid x)_{H}=P \Delta(x) .
$$

A morphism $f: C \rightarrow D$ of quadratic pair modules is given by three homomorphisms $f_{i}: C_{i} \rightarrow D_{i}, i=0,1$,ee, commuting with the structure maps, i.e. $f_{0} \partial=\partial f_{1}, f_{1} P=P f_{e e}, f_{e e} H=H f_{0}$. Morphisms of quadratic pair modules are also compatible with $T, \Delta$, and $\cdot \eta$. A quasi-isomorphism is a morphism inducing isomorphisms in $h_{0}$ and $h_{1}$.

\section{QuAdratiC PAIR ALGEBRAS}

In this section we recall the nature of the quadratic algebraic models for ring spectra constructed in [BM06a and we prove Theorem 1.3.

A quadratic pair algebra is an $\mathbb{N}$-graded quadratic pair module $B=\left\{B_{n, *}\right\}_{n \in \mathbb{N}}$, together with multiplications, $n, m \in \mathbb{N}$,

$$
\begin{aligned}
& B_{n, 0} \times B_{m, 0} \stackrel{\dot{ }}{\longrightarrow} B_{n+m, 0}, \\
& B_{n, 0} \times B_{m, 1} \longrightarrow B_{n+m, 1}, \\
& B_{n, 1} \times B_{m, 0} \longrightarrow B_{n+m, 1}, \\
& B_{n, e e} \times B_{m, e e} \longrightarrow B_{n+m, e e},
\end{aligned}
$$

and an element $1 \in B_{0,0}$ with $H(1)=0$ which is a (two-sided) unit for the first three multiplications and such that $(1 \mid 1)_{H} \in B_{0, e e}$ is a (two-sided) unit for the fourth multiplication. These multiplications are associative in all possible ways. 
Moreover, the following lists of equations are satisfied for $x, x_{i} \in B_{*, 0}, s, s_{i} \in B_{*, 1}$ and $a_{i} \in B_{*, e e}$. The multiplications $\cdot$ are always right linear

$$
\begin{aligned}
x_{1} \cdot\left(x_{2}+x_{3}\right) & =x_{1} \cdot x_{2}+x_{1} \cdot x_{3}, \\
x \cdot\left(s_{1}+s_{2}\right) & =x \cdot s_{1}+x \cdot s_{2}, \\
s \cdot\left(x_{1}+x_{2}\right) & =s \cdot x_{1}+s \cdot x_{2}, \\
a_{1} \cdot\left(a_{2}+a_{3}\right) & =a_{1} \cdot a_{2}+a_{1} \cdot a_{3} .
\end{aligned}
$$

The multiplications $\cdot$ satisfy the following left distributivity laws

$$
\begin{aligned}
\left(x_{1}+x_{2}\right) \cdot x_{3} & =x_{1} \cdot x_{3}+x_{2} \cdot x_{3}+\partial P\left(\left(x_{2} \mid x_{1}\right)_{H} \cdot H\left(x_{3}\right)\right), \\
\left(x_{1}+x_{2}\right) \cdot s & =x_{1} \cdot s+x_{2} \cdot s+P\left(\left(x_{2} \mid x_{1}\right)_{H} \cdot H \partial(s)\right), \\
\left(s_{1}+s_{2}\right) \cdot x & =s_{1} \cdot x+s_{2} \cdot x+P\left(\left(\partial\left(s_{2}\right) \mid \partial\left(s_{1}\right)\right)_{H} \cdot H(x)\right), \\
\left(a_{1}+a_{2}\right) \cdot a_{3} & =a_{1} \cdot a_{3}+a_{2} \cdot a_{3} .
\end{aligned}
$$

The homomorphisms $\partial$ are compatible with the multiplications · in the following sense

$$
\begin{aligned}
\partial(x \cdot s) & =x \cdot \partial(s), \\
\partial(s \cdot x) & =\partial(s) \cdot x, \\
\partial\left(s_{1}\right) \cdot s_{2} & =s_{1} \cdot \partial\left(s_{2}\right) .
\end{aligned}
$$

And finally, we have compatibility conditions for the multiplications $\cdot$ and the maps $P, H, \Delta$, and $(-\mid-)_{H}$,

$$
\begin{aligned}
P\left((x \mid x)_{H} \cdot a\right)= & x \cdot P(a), \\
P(a \cdot \Delta(x))= & P(a) \cdot x, \\
H\left(x_{1} \cdot x_{2}\right)= & \left(x_{1} \mid x_{1}\right)_{H} \cdot H\left(x_{2}\right)+H\left(x_{1}\right) \cdot \Delta\left(x_{2}\right), \\
H \partial P\left(a_{1} \cdot a_{2}\right)= & H \partial P\left(a_{1}\right) \cdot a_{2}+a_{1} \cdot H \partial P\left(a_{2}\right) \\
& -H \partial P\left(a_{1}\right) \cdot H \partial P\left(a_{2}\right), \\
\left(x_{1} \cdot x_{2} \mid x_{3} \cdot x_{4}\right)_{H}= & \left(x_{1} \mid x_{3}\right)_{H} \cdot\left(x_{2} \mid x_{4}\right)_{H} .
\end{aligned}
$$

Ungraded quadratic pair algebras were first considered in BJP06 in order to represent classes in third Mac Lane cohomology. The graded notion, which is the one we mainly use in this paper, was introduced in BM06a.

A morphism of quadratic pair algebras is a morphism of graded quadratic pair modules which preserves the products . A quasi-isomorphism is a morphism inducing isomorphisms in $h_{0}$ and $h_{1}$.

For $B$ a quadratic pair algebra $h_{0} B$ is a ring ( $\mathbb{N}$-graded) and $h_{1} B$ is an $h_{0} B$ bimodule in a natural way. Moreover, the $k$-nvariant

$$
\text { - } \eta: h_{0} B \longrightarrow h_{1} B
$$

is an $h_{0} B$-bimodule homomorphism by (A4/A5/A8).

The relations in the following lemma are consequences of (A2).

Lemma 3.2. With the notation above the following equations hold.

(1) $0 \cdot x_{2}=0$,

(2) $\left(-x_{1}\right) \cdot x_{2}=-x_{1} \cdot x_{2}+\partial P\left(\left(x_{1} \mid x_{1}\right)_{H} \cdot H\left(x_{2}\right)\right)$,

(3) $(-x) \cdot s=-x \cdot s+P\left((x \mid x)_{H} \cdot H \partial(s)\right)$.

Definition 3.3. Let $B$ be a quadratic pair algebra. Given elements $a, b, c \in h_{0} B$, of degree $p, q, r \in \mathbb{N}$ with $a b=0$ and $b c=0$ the Massey product is the subset

$$
\langle a, b, c\rangle \subset h_{1} B_{p+q+r},
$$

which is a coset of the subgroup

$$
\left(h_{1} B_{p+q}\right) \cdot c+a \cdot\left(h_{1} B_{q+r}\right),
$$


defined as follows. Given $\bar{a} \in B_{p, 0}, \bar{b} \in B_{q, 0}, \bar{c} \in B_{r, 0}$ representing $a, b, c$, there exist $\overline{a b} \in B_{p+q, 1}, \overline{b c} \in B_{q+r, 1}$ such that $\partial(\overline{a b})=\bar{a} \cdot \bar{b}, \partial(\overline{b c})=\bar{b} \cdot \bar{c}$ and one can easily check that

$$
-\overline{a b} \cdot \bar{c}+\bar{a} \cdot \overline{b c} \in h_{1} B_{p+q+r} \subset B_{p+q+r, 1} .
$$

The coset $\langle a, b, c\rangle \subset h_{1} B_{p+q+r}$ coincides with the set of elements obtained in this way for all different choices of $\bar{a}, \bar{b}, \bar{c}, \overline{a b}$ and $\overline{b c}$.

In BM06a we prove the following theorem as a main result.

Theorem 3.4 ([BM06a, Theorem 6.4]). There is a functor

$$
\pi_{*, *}: \text { (connective ring spectra) } \longrightarrow \text { (quadratic pair algebras) }
$$

together with natural isomorphisms

$$
\begin{aligned}
h_{0} \pi_{*, *} R \cong \pi_{*} R, \text { of rings, } \\
h_{1} \pi_{*, *} R \cong \Sigma^{-1} \pi_{*} R, \text { of bimodules, }
\end{aligned}
$$

such that the Massey products in $\pi_{*, *} R$ coincide with the Toda brackets in $\pi_{*} R$. Moreover, using the isomorphisms as identifications the algebraically-defined $k$ invariant of the quadratic pair algebra $\pi_{*, *} R$

$$
\cdot \eta: \pi_{*} R \otimes \mathbb{Z} / 2 \longrightarrow \Sigma^{-1} \pi_{*} R,
$$

coincides with the multiplication by the image of the stable Hopf map under the homomorphism $\pi_{*} S \rightarrow \pi_{*} R$ induced by the unit $S \rightarrow R$.

Theorem 1.3 will then follow from the following one.

Theorem 3.5. If $B$ is a quadratic pair algebra then the $k$-invariant (3.1) and the Massey products in Definition 3.3 endow $h_{0} B$ with the structure of a ring with secondary operations with coefficients in $h_{1} B$ in the sense of Definition 1.2.

For the sake of simplicity in the proof of Theorem 3.5 we will use assume that $B$ satisfies the property $(\mathrm{H})$.

(H) Any element in $x \in h_{0} B$ is the image of an element $\bar{x} \in B_{*, 0}$ with $H(\bar{x})=0$.

This property is not unusual. For instance, given a ring spectrum $R$ the quadratic pair algebra $\pi_{*, *} R$ defined by Theorem 3.4 satisfies property $(\mathrm{H})$. Indeed the following lemma holds.

Lemma 3.6. Given a quadratic pair algebra $B$ there is another one $\widehat{B}$ satisfying property $(H)$ and a natural quasi-isomorphism $B \rightarrow \widehat{B}$.

Here $\widehat{B}$ is a fibrant replacement of $B$ in the cofibration category of quadratic pair algebras and is obtained "attaching cells" to $B$. We will not discuss the homotopical aspects of quadratic pair algebras in this paper, so we leave the proof of Lemma 3.6 to the interested reader. This lemma shows that there is no loss of generality if we only prove Theorem 3.5 for quadratic pair algebras satisfying property $(\mathrm{H})$.

Remark 3.7. Before beginning the proof of Theorem 3.5 we want to remark that in orther to check the inclusions and equalities in Definition 1.2 it is enough to check that the brackets have an alement in common. Then the inclusion (resp. equality) follows from the obvious analogous inclusion (resp. equality) between the indeterminacies which is clear in all cases. The same applies to the proof of Theorem 6.4. 
Proof of Theorem 3.5. We assume that all representatives chosen in $B_{*, 0}$ are in Ker $H$. Let us check that equations (T1)-(T6) hold.

(T1) If $a=0$ we can take $\bar{a}=0$ and $\overline{a b}=0$ so $-\overline{a b} \cdot \bar{c}+\bar{a} \cdot \overline{b c}=0$. Similarly in the other two cases.

(T2) We can take $\overline{a+a^{\prime}}=\bar{a}+\bar{a}^{\prime}$ and by (A2) we can also take $\overline{\left(a+a^{\prime}\right) b}=\overline{a b}+\overline{a^{\prime} b}$, therefore

$$
\begin{array}{r}
-\overline{\left(a+a^{\prime}\right) b} \cdot \bar{c}+\overline{a+a^{\prime}} \cdot \overline{b c} \stackrel{\overline{\mathrm{A} 2}=}{=}-\overline{a^{\prime} b} \cdot \bar{c}-\overline{a b} \cdot \bar{c}+\bar{a} \cdot \overline{b c}+\bar{a}^{\prime} \cdot \overline{b c}-P(\left(a^{\prime} \mid a\right)_{H} \cdot \underbrace{H(\bar{b} \cdot \bar{c})}_{(\underline{\mathrm{A} 6})=0}) \\
\quad=-\overline{a^{\prime} b} \cdot \bar{c}+\bar{a} \cdot \overline{b c}-\overline{a b} \cdot \bar{c}+\bar{a}^{\prime} \cdot \overline{b c}-\underbrace{P(\bar{a} \cdot \bar{b} \cdot \bar{c} \mid \bar{a} \cdot \bar{b} \cdot \bar{c})_{H}}_{=a \cdot b \cdot c \cdot \eta=0} .
\end{array}
$$

One proceeds similarly with the two other variables.

(T3) By (A33) $\partial(\bar{a} \cdot \overline{b c})=\bar{a} \cdot \bar{b} \cdot \bar{c}$, hence the first equation in (T3) follows from

$$
\bar{a} \cdot(-\overline{b c} \cdot \bar{d}+\bar{b} \cdot \overline{c d}) \stackrel{\text { Al} 1}{=}-(\bar{a} \cdot \overline{b c}) \cdot \bar{d}+(\bar{a} \cdot \bar{b}) \cdot \overline{c d} .
$$

The second one follows similarly.

(T4) By (A3) $\partial(\bar{b} \cdot \overline{c d})=\bar{b} \cdot \bar{c} \cdot \bar{d}$ therefore $-\overline{a b c} \cdot \bar{d}+\bar{a} \cdot \bar{b} \cdot \overline{c d}$ lies in both $\langle a b, c, d\rangle$ and $\langle a, b c, d\rangle$. Similarly the element $-\overline{a c} \cdot \bar{c} \cdot \bar{d}+\bar{a} \cdot \overline{b c d}$ belongs to the other two Massey products.

(T5) This follows from

$$
\begin{aligned}
& (-\overline{a b} \cdot \bar{c}+\bar{a} \cdot \overline{b c}) \cdot \bar{d}+\bar{a} \cdot(-\overline{b c} \cdot \bar{d}+\bar{b} \cdot \overline{c d}) \stackrel{\text { A1|A2 }}{=}-\overline{a b} \cdot \bar{c} \cdot \bar{d}+\bar{a} \cdot \overline{b c} \cdot \bar{d} \\
& -\bar{a} \cdot \overline{b c} \cdot \bar{d}+\bar{a} \cdot \bar{b} \cdot \overline{c d} \\
& =\quad-\overline{a b} \cdot \bar{c} \cdot \bar{d}+\bar{a} \cdot \bar{b} \cdot \overline{c d} \\
& =-\overline{a b} \cdot \partial(\overline{c d})+\partial(\overline{a b}) \cdot \overline{c d} \\
& \text { A3 }=0 .
\end{aligned}
$$

Finally (T6) follows from [BM06a, Proposition 6.6].

\section{Modules over quadratic Pair algebras}

The quadratic algebraic models of module spectra leading to Theorem 1.6 are as follows.

Let $B$ be a quadratic pair algebra. A right $B$-module is an $\mathbb{N}$-graded quadratic pair module $M=\left\{M_{n, *}\right\}_{n \in \mathbb{N}}$ together with multiplications, $n, m \geq 0$,

$$
\begin{aligned}
& M_{n, 0} \times B_{m, 0} \longrightarrow M_{n+m, 0}, \\
& M_{n, 0} \times B_{m, 1} \stackrel{\longrightarrow}{\longrightarrow} M_{n+m, 1} \text {, } \\
& M_{n, 1} \times B_{m, 0} \stackrel{\longrightarrow}{\longrightarrow} M_{n+m, 1} \text {, }
\end{aligned}
$$

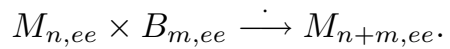

These multiplications are associative with respect to the multiplications in $B$. Moreover, $1 \in B_{0,0}$ acts trivially on $M_{*, 0}$ and $M_{*, 1}$, and $(1 \mid 1)_{H} \in B_{0, e e}$ acts trivially on $M_{*, e e}$. Furthermore, equations (A1)-(A8) hold when we replace the elements on the left of any multiplication - by elements in $M$.

If $M$ is a right $B$-module then $h_{0} M$ and $h_{1} M$ are right $h_{0} B$-modules and there is a natural right $h_{0} B$-module homomorphism

$$
h_{0} M \otimes_{h_{0} B} h_{1} B \stackrel{\cdot}{\longrightarrow} h_{1} M,
$$

see BM06a, 7], extending the $k$-invariant since $x \cdot \eta=x \cdot P(1 \mid 1)_{H}$ for $x \in h_{0} M$ by (A4). The $k$-invariant of a right $B$-module $M$ is a right $h_{0} B$-module homomorphism by A4[A5|A 8 . 
Definition 4.2. Given a right $B$-module $M$ and elements $a \in h_{0} M, b, c \in h_{0} B$, of degree $p, q, r$, such that $a b=0$ and $b c=0$ there is defined a Massey product

$$
\langle a, b, c\rangle \subset h_{1} M_{p+q+r}
$$

by the same procedure as in Definition 3.3 which is a coset of

$$
\left(h_{1} M_{p+q}\right) \cdot c+a \cdot\left(h_{1} B_{q+r}\right) .
$$

We show the following theorem in BM06a].

Theorem 4.3 (BM06a, Theorem 7.4]). Let $R$ be a connective ring spectrum. There is a functor

$$
\left.\left.\pi_{*, *}: \text { (connective right } R \text {-modules }\right) \longrightarrow \text { (right }\left(\pi_{*, *} R\right) \text {-modules }\right) .
$$

Here the quadratic pair algebra $\pi_{*, *} R$ is obtained by using the functor in Theorem 3.4. Moreover, if we use the isomorphisms in Theorem 3.4 as identifications then for any right $R$-module $K$ there are natural isomorphisms of right $\pi_{*} R$-modules

$$
\begin{aligned}
& h_{0} \pi_{*, *} K \cong \pi_{*} K, \\
& h_{1} \pi_{*, *} K \cong \Sigma^{-1} \pi_{*} K .
\end{aligned}
$$

Using these isomorphisms as identifications the algebraically-defined homomorphism (4.1) associated to $\pi_{*, *} K$

$$
\cdot: \pi_{*} K \otimes_{\pi_{*} R} \Sigma^{-1} \pi_{*} R \longrightarrow \Sigma^{-1} \pi_{*} K
$$

is defined by the right right $\pi_{*} R$-module structure of $\pi_{*} K$ according to the formula

$$
m \cdot\left(\Sigma^{-1} a\right)=(-1)^{|m|} \Sigma^{-1}(m \cdot a) .
$$

In particular the $k$-invariant of $\pi_{*, *} K$ coincides with the multiplication by the stable Hopf map $\eta$. Furthermore, Massey products in $\pi_{*, *} K$ coincide with Toda brackets in $\pi_{*} K$.

Now Theorem 1.6 follows from the following theorem.

Theorem 4.4. If $B$ is a quadratic pair algebra and $M$ is a right $B$-module then (4.1) and the Massey products in Definition 4.2 endow $h_{0} M$ with the structure of a module with secondary operations with coefficients in $h_{1} M$ in the sense of Definition 1.5 .

The proof is completely analogous to the proof of Theorem 3.5 so we leave it to the reader.

\section{Symmetric TRACK GROUPS}

In order to describe the quadratic algebraic models associated to commutative ring spectra we need to endow quadratic pair modules with symmetries coming from the action of sign groups as we describe in this section.

A sign group is a diagram in the category of groups

$$
\{ \pm 1\} \stackrel{\iota}{\hookrightarrow} G_{\square} \stackrel{\delta}{\rightarrow} G \stackrel{\varepsilon}{\longrightarrow}\{ \pm 1\}
$$

where the first two morphisms form an extension. By abuse of notation we denote this sign group just by $G_{\square}$. The group law of the groups defining a sign group is denoted multiplicatively.

Given a sign group $G_{\square}$ the "group ring" $A\left(G_{\square}\right)$ is the ungraded quadratic pair algebra with generators

- $[g]$ for any $g \in G$ on the 0 -level,

- $[t]$ for any $t \in G_{\square}$ on the 1-level,

- no generators on the ee-level, 
satisfying the following relations for $g, h \in G, s, t \in G_{\square}$ and $\omega=\imath(-1)$.

$$
\begin{aligned}
H[g] & =0, \\
{[1] } & =1 \text { for } 1 \in G, \\
{[g h] } & =[g] \cdot[h], \\
\partial[t] & =-[\delta(t)]+\varepsilon \delta(t), \\
{[s t] } & =[\delta(s)] \cdot[t]+\varepsilon \delta(t) \cdot[s]+\left(\begin{array}{c}
\varepsilon \delta(s) \\
2
\end{array}\right)\left(\begin{array}{c}
\varepsilon \delta(t) \\
2
\end{array}\right) P(1 \mid 1)_{H}, \\
{[\omega] } & =P(1 \mid 1)_{H} .
\end{aligned}
$$

Sign groups were introduced in BM06c, and the "group ring" of a sign group was first considered in [BM06b].

The following lemma follows easily from (S1]S3) and the fact that $H$ is quadratic.

Lemma 5.1. Given $t \in G_{\square}$ the following equation holds.

$$
H \partial[t]=([\delta(t)] \mid-\partial[t])_{H}+\left(\begin{array}{c}
\varepsilon \delta(t) \\
2
\end{array}\right)(1 \mid 1)_{H} .
$$

The following useful relation follows from (S2/S5).

Lemma 5.2. For $1 \in G_{\square}$ we have $[1]=0$.

The action of $A_{0}\left(G_{\square}\right)$ on the left of $A_{1}\left(G_{\square}\right)$ is dertermined by relation (S5) in terms of the group structure of $G_{\square}$ since $\delta$ is surjective. The right action is given by the following lemma.

Lemma 5.3. For $s, t \in G_{\square}$ the following relation holds in $A\left(G_{\square}\right)$.

$$
[s t]=[s] \cdot[\delta(t)]+\varepsilon \delta(s) \cdot[t] .
$$

Proof. On one hand by (S4|A1]3.2.3)

$$
[s] \cdot \partial[t]=-[s] \cdot[\delta(t)]+\varepsilon \delta(t) \cdot[s]-\left(\begin{array}{c}
\varepsilon \delta(t) \\
2
\end{array}\right) P H \partial[s] .
$$

On the other hand by (S4|A2 $3.2,35.1|\bar{A} 8| \mathrm{M} 3|\mathrm{~A} 3|$

$$
\begin{aligned}
\partial[s] \cdot[t]= & -[\delta(s)] \cdot[t]+\varepsilon \delta(s) \cdot[t]+P\left((-\partial[s] \mid[\delta(s)])_{H} \cdot H \partial[t]\right) \\
= & -[s] \cdot[\delta(t)]-[\delta(s)] \cdot[t]+[s] \cdot[\delta(t)]+\varepsilon \delta(s) \cdot[t] \\
& +\left(\begin{array}{c}
\varepsilon \delta(t) \\
2
\end{array}\right) P(-\partial[s] \mid[\delta(s)])_{H} .
\end{aligned}
$$

By (A3) $\partial[s] \cdot[t]=[s] \cdot \partial[t]$, hence by using the two previous equations together with (5.1) one obtains

$$
[\delta(s)] \cdot[t]+\varepsilon \delta(t) \cdot[s]+\left(\begin{array}{c}
\varepsilon \delta(s) \\
2
\end{array}\right)\left(\begin{array}{c}
\varepsilon \delta(t) \\
2
\end{array}\right) P(1 \mid 1)_{H}=[s] \cdot[\delta(t)]+\varepsilon \delta(s) \cdot[t] .
$$

Now the lemma follows from (S5).

The homology of "group rings" of sign groups can be easily computed.

Lemma 5.4. There are natural isomorphisms

$$
\begin{aligned}
h_{0} A\left(G_{\square}\right) & \cong \mathbb{Z}, \\
h_{1} A\left(G_{\square}\right) & \cong \mathbb{Z} / 2 .
\end{aligned}
$$

The first one is induced by $[g] \mapsto \varepsilon(g)$, and $h_{1} A\left(G_{\square}\right)$ is generated by $[\omega]$. 
The main examples of sign groups are the symmetric track groups $\operatorname{Sym}_{\square}(n)$ associated to the sign homomorphism of the symmetric groups

$$
\varepsilon=\operatorname{sign}: \operatorname{Sym}(n) \rightarrow\{ \pm 1\} .
$$

The group $\operatorname{Sym}_{\square}(n)$ has a presentation with generators $\omega, t_{i}, 1 \leq i \leq n-1$, and relations

$$
\begin{aligned}
t_{i}^{2} & =1 \text { for } 1 \leq i \leq n-1, \\
\left(t_{i} t_{i+1}\right)^{3} & =1 \text { for } 1 \leq i \leq n-2, \\
\omega^{2} & =1 \\
t_{i} \omega & =\omega t_{i} \text { for } 1 \leq i \leq n-1, \\
t_{i} t_{j} & =\omega t_{j} t_{i} \text { for } 1 \leq i<j-1 \leq n-1 .
\end{aligned}
$$

Moreover, the structure of sign group is given by $\imath(-1)=\omega, \delta(\omega)=0$, and $\delta\left(t_{i}\right)=$ $(i i+1)$, the permutation exchanging $i$ and $i+1$ in $\{1, \ldots, n\}$.

Below we use the homomorphisms

$$
\begin{aligned}
& S^{n} \wedge-: \operatorname{Sym}_{\square}(m) \longrightarrow \operatorname{Sym}_{\square}(n+m), \\
& -\wedge S^{m}: \operatorname{Sym}_{\square}(n) \longrightarrow \operatorname{Sym}_{\square}(n+m),
\end{aligned}
$$

defined on generators by

$$
\begin{aligned}
t_{i} \wedge S^{m} & =t_{i}, \quad 1 \leq i \leq n-1, \\
\omega \wedge S^{m} & =\omega, \\
S^{n} \wedge t_{i} & =t_{n+i}, \quad 1 \leq i \leq m-1, \\
S^{n} \wedge \omega & =\omega .
\end{aligned}
$$

These homomorphisms are related by the following formula.

Lemma 5.6. Let $\tau_{n, m} \in \operatorname{Sym}(n+m)$ be the permutation exchanging the first block of $n$ elements with the last block of $m$ elements and let $\hat{\tau}_{n, m} \in \operatorname{Sym}_{\square}(n+m)$ be an element with $\delta\left(\hat{\tau}_{n, m}\right)=\tau_{n, m}$. Then for any $t \in \operatorname{Sym}_{\square}(n)$ we have

$$
\left(S^{m} \wedge t\right) \hat{\tau}_{n, m}=\hat{\tau}_{n, m}\left(t \wedge S^{m}\right) \omega^{n m\left(\begin{array}{c}
\varepsilon_{2}(t) \\
2
\end{array}\right) .}
$$

Notice that Lemma 5.6 does not depend on the choice of $\hat{\tau}_{n, m}$ since the two possible choices differ in $\omega$, which is central. For the proof of Lemma 5.6 we choose

$$
\hat{\tau}_{n, m}=\overbrace{\underbrace{\overbrace{m} \cdots t_{1}}_{m \text { generators }} \cdots \cdots \cdot \underbrace{n \text { groups of }}_{m \text { generators }} m \text { generators }}^{t_{n+m-1} \cdots t_{n}} .
$$

Proof of Lemma 5.6. The equation holds for $t=\omega$, which is central, therefore we can restrict to the case $t=t_{i}, 1 \leq i \leq n-1$. We check by induction in $j$ that

$$
t_{i+j} \cdots t_{i+1} t_{i} t_{i+1} \cdots t_{i+j}=t_{i} \cdots t_{i+j-1} t_{i+j} t_{i+j-1} \cdots t_{i} .
$$

For $j=1$ this is follows from (5.5), and if it is true for $j-1$ then

$$
\begin{aligned}
t_{i+j} t_{i+j-1} \cdots t_{i+1} t_{i} t_{i+1} \cdots t_{i+j-1} t_{i+j} & =t_{i+j} t_{i} \cdots t_{i+j-2} t_{i+j-1} t_{i+j-2} \cdots t_{i} t_{i+j} \\
& =t_{i} \cdots t_{i+j-2} t_{i+j} t_{i+j-1} t_{i+j} t_{i+j-2} \cdots t_{i} \\
& =t_{i} \cdots t_{i+j-2} t_{i+j-1} t_{i+j} t_{i+j-1} t_{i+j-2} \cdots t_{i} .
\end{aligned}
$$

Equation (a) is equivalent to

$$
t_{i+j-1} \cdots t_{i} t_{i+j} \cdots t_{i+1} t_{i}=t_{i+j} t_{i+j-1} \cdots t_{i} t_{i+j} \cdots t_{i+1} .
$$

One can now easily check by using the other relations of the symmetric track groups that (b) for $j=m$ implies

$$
\hat{\tau}_{n, m} t_{i}=t_{i+m} \hat{\tau}_{n, m} \omega^{m(n-2)},
$$


hence the lemma follows.

Remark 5.8. The symmetric track groups were defined in [BM06c, 5] in a geometric way in terms of tracks. In [BM06c, 6] we relate them to the positive pin group, obtaining in this way the presentation above, see [BM06c, Theorem 6.11]. The homomorphisms $S^{n} \wedge-$ and $-\wedge S^{m}$ were geometrically defined in [BM06b, 8]. We also give formulas for $S^{n} \wedge$ - in terms of the positive pin group in BM06b, 17], from wich we derive the formulas for $S^{n} \wedge-$ in terms of the presentation. The formulas for $-\wedge S^{m}$ in terms of the presentation follow then from the definition of $-\wedge S^{m}$ in [BM06b, 8] and from Lemma 5.6.

The next lemma encodes some relevant properties of the choices in (5.7).

Lemma 5.9. The following equations hold for the elements in (5.7).

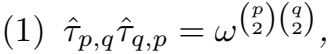

(2) $\left(S^{r} \wedge \hat{\tau}_{p, s} \wedge S^{q}\right)\left(S^{r+p} \wedge \hat{\tau}_{q, s}\right)\left(\hat{\tau}_{p, r} \wedge S^{q+s}\right)\left(S^{p} \wedge \hat{\tau}_{q, r} \wedge S^{s}\right)=$ $\hat{\tau}_{p+q, r+s} \omega^{r s}\left(\left(\begin{array}{c}p \\ 2\end{array}\right)+\left(\begin{array}{c}q \\ 2\end{array}\right)+p q\right)$.

The proof only uses the presentation of the symmetric track groups as in Lemma 5.6. We leave it to the reader.

Relation (S5) and Lemmas 5.3 and 5.6 yield the following result.

Lemma 5.10. With the notation of Lemma[5.6 the equation

$$
\left[S^{m} \wedge t\right] \cdot\left[\tau_{n, m}\right]=\left[\tau_{n, m}\right] \cdot\left[t \wedge S^{m}\right]
$$

holds.

We also use below the well-known cross product homomorphisms

$$
\operatorname{Sym}(n) \times \operatorname{Sym}(m) \longrightarrow \operatorname{Sym}(n+m):(\sigma, \tau) \mapsto \sigma \times \tau .
$$

Here $\sigma \times \tau$ permutes the first $n$ elements $\{1, \ldots, n\}$ of $\{1, \ldots, n, n+1, \ldots, n+m\}$ according to $\sigma$ and the last $m$ elements $\{n+1, \ldots, n+m\}$ according to $\tau$. These homomorphisms satisfy $\tau_{n, m}(\sigma \times \tau)=(\tau \times \sigma) \tau_{n, m}$. Moreover, if $1_{m} \in \operatorname{Sym}(m)$ denotes the unit of the symmetric group then $\delta\left(S^{m} \wedge t\right)=1_{m} \times \delta(t)$ and $\delta\left(t \wedge S^{m}\right)=$ $\delta(t) \times 1_{m}$.

\section{6. $E_{\infty}$-QUADRATIC PAIR ALGEBRAS}

An $E_{\infty}$-quadratic pair algebra is a quadratic pair algebra $B$ together with a cup-one product operation

$$
\smile_{1}: B_{n, 0} \times B_{m, 0} \longrightarrow B_{n+m, 1}, \quad n, m \geq 0,
$$

such that the quadratic pair module $B_{n, *}$ is a right $A\left(\operatorname{Sym}_{\square}(n)\right)$-module and the following compatibility conditions hold. Let $x_{i} \in B_{n_{i}, 0}, s_{i} \in B_{n_{i}, 1}, a_{i} \in B_{n_{i}, e e}$, $g_{i}, g_{i}^{\prime} \in \operatorname{Sym}\left(n_{i}\right)$, and $r_{i} \in \operatorname{Sym}_{\square}\left(n_{i}\right)$. The product in the quadratic pair algebra $B$ is equivariant with respect to the right $A\left(\operatorname{Sym}_{\square}(n)\right)$-module structures in the following way

$$
\begin{aligned}
\left(x_{1} \cdot\left[g_{1}\right]\right) \cdot\left(x_{2} \cdot\left[g_{2}\right]\right) & =\left(x_{1} \cdot x_{2}\right) \cdot\left[g_{1} \times g_{2}\right], \\
\left(s_{1} \cdot\left[g_{1}\right]\right) \cdot\left(x_{2} \cdot\left[g_{2}\right]\right) & =\left(s_{1} \cdot x_{2}\right) \cdot\left[g_{1} \times g_{2}\right], \\
\left(a_{1} \cdot\left(\left[g_{1}\right] \mid\left[g_{1}^{\prime}\right]\right)_{H}\right) \cdot\left(a_{2} \cdot\left(\left[g_{2}\right] \mid\left[g_{2}^{\prime}\right]\right)_{H}\right) & =\left(a_{1} \cdot a_{2}\right) \cdot\left(\left[g_{1} \times g_{2}\right] \mid\left[g_{1}^{\prime} \times g_{2}^{\prime}\right]\right)_{H}, \\
x_{1} \cdot\left(x_{2} \cdot\left[r_{2}\right]\right) & =\left(x_{1} \cdot x_{2}\right) \cdot\left[S^{n_{1}} \wedge r_{2}\right] .
\end{aligned}
$$

The cup-one product measures the lack of commutativity, i.e. if $\tau_{p, q} \in \operatorname{Sym}(p+q)$ denotes the permutation exchanging the blocks $\{1, \ldots, p\}$ and $\{p+1, \ldots, p+q\}$, 
$p, q \geq 0$, then

$$
\begin{aligned}
&\left(x_{2} \cdot x_{1}\right) \cdot\left[\tau_{n_{1}, n_{2}}\right]+\partial\left(x_{1} \smile_{1} x_{2}\right)=x_{1} \cdot x_{2}+\partial P\left(H\left(x_{2}\right) \cdot T H\left(x_{1}\right)\right) \cdot\left[\tau_{n_{1}, n_{2}}\right], \\
&\left(x_{2} \cdot s_{1}\right) \cdot\left[\tau_{n_{1}, n_{2}}\right]+\partial\left(s_{1}\right) \smile_{1} x_{2}=s_{1} \cdot x_{2}+P\left(H\left(x_{2}\right) \cdot T H \partial\left(s_{1}\right)\right) \cdot\left[\tau_{n_{1}, n_{2}}\right] .
\end{aligned}
$$

The cup-one product is itself commutative in the following sense

$$
\begin{aligned}
\left(x_{2} \smile_{1} x_{1}\right) \cdot\left[\tau_{n_{1}, n_{2}}\right]+x_{1} \smile_{1} x_{2}= & -P\left(T H\left(x_{1}\right) \cdot H\left(x_{2}\right)\right) \\
& +P\left(H\left(x_{2}\right) \cdot T H\left(x_{1}\right)\right) \cdot\left[\tau_{n_{1}, n_{2}}\right] .
\end{aligned}
$$

Let $1_{n} \in \operatorname{Sym}(n)$ be the unit element. The cup-one product also satisfies the following rules with respect to addition

$$
\begin{aligned}
x_{1} \smile_{1}\left(x_{2}+x_{3}\right)= & x_{1} \smile_{1} x_{2}+x_{1} \smile_{1} x_{3} \\
& +P\left(\partial\left(x_{1} \smile_{1} x_{2}\right) \mid\left(x_{3} \cdot x_{1}\right) \cdot\left[\tau_{n_{1}, n_{3}}\right]\right)_{H},
\end{aligned}
$$

multiplication

$$
\begin{aligned}
\left(x_{1} \cdot x_{2}\right) \smile_{1} x_{3}= & \left(\left(x_{1} \smile_{1} x_{3}\right) \cdot x_{2}\right) \cdot\left[1_{n_{1}} \times \tau_{n_{2}, n_{3}}\right]+x_{1} \cdot\left(x_{2} \smile_{1} x_{3}\right) \\
& +P\left(\left(\partial\left(x_{1} \smile_{1} x_{3}\right) \mid\left(x_{3} \cdot x_{1}\right) \cdot\left[\tau_{n_{1}, n_{3}}\right]\right)_{H} \cdot H\left(x_{2}\right)\right) \cdot\left[1_{n_{1}} \times \tau_{n_{2}, n_{3}}\right] \\
& +P\left(H\left(x_{3}\right) \cdot\left(x_{1} \mid x_{1}\right)_{H} \cdot T H\left(x_{2}\right)\right) \cdot\left[\tau_{n_{1}+n_{2}, n_{3}}\right] \\
& -P\left(\left(x_{1} \mid x_{1}\right)_{H} \cdot H\left(x_{3}\right) \cdot T H\left(x_{2}\right)\right) \cdot\left[1_{n_{1}} \times \tau_{n_{2}, n_{3}}\right],
\end{aligned}
$$

and symmetric group action

$$
\left(x_{1} \cdot\left[g_{1}\right]\right) \smile_{1}\left(x_{2} \cdot\left[g_{2}\right]\right)=\left(x_{1} \smile_{1} x_{2}\right) \cdot\left[g_{1} \times g_{2}\right] .
$$

If $B$ is an $E_{\infty}$-quadratic pair algebra then $h_{0} B$ is a commutative ring and $h_{1} B$ is an $h_{0} B$-module, see BM06a, Lemma 9.9].

Appart from Massey products the homology of an $E_{\infty}$-quadratic pair algebra is endowed with the following secondary operation.

Definition 6.1. Let $B$ be an $E_{\infty}$-quadratic pair algebra. Given an element $a \in$ $h_{0} B_{2 n, *}$ we define the cup-one square of $a$

$$
S q_{1}(a) \in h_{1} B_{4 n, *}
$$

in the following way. Choose a representative $\bar{a} \in B_{2 n, 0}$ of $a$ and an element in the symmetric track group $\hat{\tau} \in \operatorname{Sym}_{\square}(4 n)$ whose boundary is the shuffle permutation $\delta(\hat{\tau})=\tau_{2 n, 2 n}$. Then

$$
S q_{1}(a)=-\bar{a}^{2} \cdot[\hat{\tau}]+\bar{a} \smile_{1} \bar{a}-P(H(\bar{a}) \cdot T H(\bar{a})) \cdot\left[\tau_{2 n, 2 n}\right] \in h_{1} B_{4 n, *} \cdot
$$

We leave it to the reader to check that the cup-one square does not depend on the choice of $\bar{a}$. However it does depend on the choice of $\hat{\tau}$. There are two possible choices, namely $\hat{\tau}$ and $\omega \hat{\tau}$. The difference between the two possible cup-one squares is computed in the following lemma.

Lemma 6.2 ([BM06a, Lemma 9.11]). Let $S q_{1}$ be cup-one square in an $E_{\infty}$-quadratic pair algebra $B$ associated to the lift $\hat{\tau}$ of the shuffle permutation and let $S q_{1}^{\omega}$ be the cup-one square associated to $\omega \hat{\tau}$. Then given $a \in h_{0} B_{2 n, *}$

$$
S q_{1}^{\omega}(a)=S q_{1}(a)+a^{2} \cdot \eta
$$

The main result in BM06a concerning $E_{\infty}$-quadratic pair algebras and ring spectra is the following. 
Theorem 6.3 ([BM06a, Theorem 9.12]). There is a commutative diagram of functors

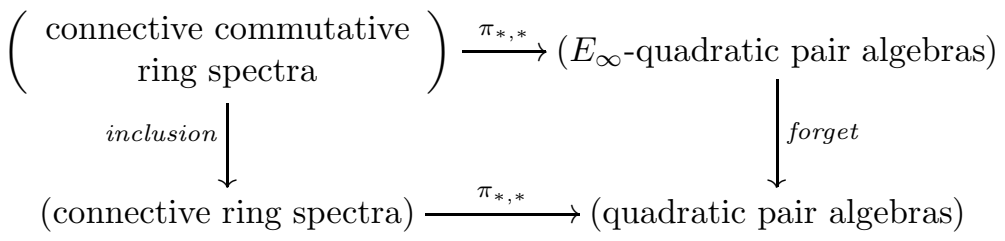

Here the lower arrow is the functor in Theorem 3.4. Moreover, for a commutative ring spectrum $R$ the algebraic cup-one squares in $\pi_{*, *} R$ correspond to the topologically-defined cup-one squares in $\pi_{*} R$.

Theorem 1.9 follows from Theorem 6.3 and from the following result.

Theorem 6.4. If $B$ is an $E_{\infty}$-quadratic pair algebra then the $k$-invariant (3.1), the Massey products in Definition 3.3, and the cup-one squares in Definition 6.1 associated to the choices of $\hat{\tau}_{2 n, 2 n}$ in (5.7) endow $h_{0} B$ with the structure of a commutative ring with commutative secondary operations with coefficients in $h_{1} B$ in the sense of Definition 1.8.

In order to prove this theorem we need a technical lemma. $E_{\infty}$-quadratic pair algebras are defined above by using a minimal set of equations. Some other useful equations are listed in the following lemma.

Lemma 6.5. With the notation above the following equations are also satisfied in the $E_{\infty}$-quadratic pair algebra $B$ for elements with $H\left(x_{i}\right)=0$.

(1) $\left(x_{1} \cdot\left[g_{1}\right]\right) \cdot\left(s_{2} \cdot\left[g_{2}\right]\right)=\left(x_{1} \cdot s_{2}\right) \cdot\left[g_{1} \times g_{2}\right]$,

(2) $\left(x_{1} \cdot\left[r_{1}\right]\right) \cdot x_{2}=\left(x_{1} \cdot x_{2}\right) \cdot\left[r_{1} \wedge S^{n_{2}}\right]$,

(3) $\left(s_{2} \cdot x_{1}\right) \cdot\left[\tau_{n_{1}, n_{2}}\right]+x_{1} \smile_{1} \partial\left(s_{2}\right)=x_{1} \cdot s_{2}$,

(4) $\left(x_{1}+x_{2}\right) \smile_{1} x_{3}=x_{1} \smile_{1} x_{3}+x_{2} \smile_{1} x_{3}+P\left(\partial\left(x_{1} \smile_{1} x_{3}\right) \mid\left(x_{3} \cdot x_{2}\right) \cdot\left[\tau_{n_{2}, n_{3}}\right]\right)_{H}$,

(5) $x_{1} \smile_{1}\left(x_{2} \cdot x_{3}\right)=\left(x_{2} \cdot\left(x_{1} \smile_{1} x_{3}\right)\right) \cdot\left[\tau_{n_{1}, n_{2}} \times 1_{n_{3}}\right]+\left(x_{1} \smile_{1} x_{2}\right) \cdot x_{3}$,

(6) $x_{1} \smile_{1}\left(x_{2} \cdot x_{3}\right)=\left(\left(x_{3} \cdot x_{1}\right) \smile_{1} x_{2}\right) \cdot\left[\tau_{n_{1}+n_{2}, n_{3}}\right]+\left(x_{1} \cdot x_{2}\right) \smile_{1} x_{3}$.

Proof. The following equations hold.

$$
\begin{array}{ll}
\left(\left(x_{1} \cdot\left[g_{1}\right]\right) \cdot(\right. & \left.\left.s_{2} \cdot\left[g_{2}\right]\right)\right) \cdot\left[\tau_{n_{2}, n_{1}}\right] \\
= & \left(s_{2} \cdot\left[g_{2}\right]\right) \cdot\left(x_{1} \cdot\left[g_{1}\right]\right)-\partial\left(s_{2} \cdot\left[g_{2}\right]\right) \smile_{1}\left(x_{1} \cdot\left[g_{1}\right]\right) \\
& +P(\underbrace{H\left(x_{1} \cdot\left[g_{1}\right]\right)}_{\text {E2 }} \cdot T H \partial\left(s_{2} \cdot\left[g_{2}\right]\right)) \cdot\left[\tau_{n_{2}, n_{1}}\right] \\
= & \left(s_{2} \cdot x_{1}\right) \cdot\left[g_{2} \times g_{1}\right]-\left(\partial\left(s_{2}\right) \smile_{1} x_{1}\right) \cdot\left[g_{2} \times g_{1}\right] \\
\text { E11A3[E6 } & \left(s_{2} \cdot x_{1}-\partial\left(s_{2}\right) \smile_{1} x_{1}\right) \cdot\left[g_{2} \times g_{1}\right] \\
\text { A22S1 } & \left(x_{1} \cdot s_{2}\right) \cdot\left[\tau_{n_{2}, n_{1}}\right] \cdot\left[g_{2} \times g_{1}\right] \\
\text { E2 } & \left(x_{1} \cdot s_{2}\right) \cdot\left[g_{1} \times g_{2}\right] \cdot\left[\tau_{n_{2}, n_{1}}\right] .
\end{array}
$$

Now we obtain (1) multiplying by $\left[\tau_{n_{1}, n_{2}}\right]$ on the right and using (S3). 
Equation (2) follows from

$$
\begin{aligned}
& \left(x_{1} \cdot x_{2}\right) \cdot\left[r_{1} \wedge S^{n_{2}}\right] \stackrel{\text { E2 }}{=} \quad\left(\left(x_{2} \cdot x_{1}\right) \cdot\left[\tau_{n_{1}, n_{2}}\right]+\partial\left(x_{1} \smile_{1} x_{2}\right)\right) \cdot\left[r_{1} \wedge S^{n_{2}}\right] \\
& \text { A2[E3] }=\left(x_{2} \cdot x_{1}\right) \cdot\left[\tau_{n_{1}, n_{2}}\right] \cdot\left[r_{1} \wedge S^{n_{2}}\right]+\partial\left(x_{1} \smile_{1} x_{2}\right) \cdot\left[r_{1} \wedge S^{n_{2}}\right] \\
& +\overbrace{P\left(\left(\partial\left(x_{1} \smile_{1} x_{2}\right) \mid\left(x_{2} \cdot x_{1}\right) \cdot\left[\tau_{n_{1}, n_{2}}\right]\right)_{H} \cdot H \partial\left[r_{1} \wedge S^{n_{2}}\right]\right)}^{=(\mathrm{a})} \\
& \underline{\text { 5.10|E1|A3|S3|A1] }}=\left(x_{2} \cdot\left(x_{1} \cdot\left[r_{1}\right]\right)\right) \cdot\left[\tau_{n_{1}, n_{2}}\right] \underbrace{ \pm}\left(x_{1} \smile_{1} x_{2}\right) \\
& \text { according to } \varepsilon \delta\left(r_{1}\right) \\
& -\left(x_{1} \cdot\left[\delta\left(r_{1}\right)\right]\right) \smile_{1} x_{2}+(\mathrm{a}) \\
& \mathrm{A} 86.54 \mathrm{~A} 3|\mathrm{~S} 3| \mathrm{A} 4 \mid \mathrm{A} 8[5.1]=\left(x_{2} \cdot\left(x_{1} \cdot\left[r_{1}\right]\right)\right) \cdot\left[\tau_{n_{1}, n_{2}}\right]+\left(x_{1} \cdot \partial\left[r_{1}\right]\right) \smile_{1} x_{2} \\
& \overline{\mathrm{E} 2[\mathrm{~A} 3}=\left(x_{1} \cdot\left[r_{1}\right]\right) \cdot x_{2} .
\end{aligned}
$$

Equation (3) follows from

$$
\begin{aligned}
& x_{1} \smile_{1} \partial\left(s_{2}\right) \stackrel{\text { E30 }}{=}-\left(\partial\left(s_{2}\right) \smile_{1} x_{1}\right) \cdot\left[\tau_{n_{1}, n_{2}}\right] \\
& \text { E22 }=-\left(-\left(x_{1} \cdot s_{2}\right) \cdot\left[\tau_{n_{2}, n_{1}}\right]+s_{2} \cdot x_{1}\right) \cdot\left[\tau_{n_{1}, n_{2}}\right] \\
& \text { A2 S1 S31 }=-\left(s_{2} \cdot x_{1}\right) \cdot\left[\tau_{n_{1}, n_{2}}\right]+x_{1} \cdot s_{2} \text {. }
\end{aligned}
$$

Equation (4) follows from

$$
\begin{aligned}
& \left(x_{1}+x_{2}\right) \smile_{1} x_{3} \stackrel{\text { E2 } 2}{=}-\left(x_{3} \smile_{1}\left(x_{1}+x_{2}\right)\right) \cdot\left[\tau_{n_{1}, n_{3}}\right] \\
& \text { E4|A2IS1 }=-\left(x_{3} \smile_{1} x_{2}\right) \cdot\left[\tau_{n_{1}, n_{3}}\right]-\left(x_{3} \smile_{1} x_{1}\right) \cdot\left[\tau_{n_{1}, n_{3}}\right] \\
& -P\left(\partial\left(x_{3} \smile_{1} x_{1}\right) \mid\left(x_{2} \cdot x_{3}\right) \cdot\left[\tau_{n_{3}, n_{1}}\right]\right)_{H} \cdot\left[\tau_{n_{1}, n_{3}}\right] \\
& \text { E3|A5|A8/S1/S3 }=x_{2} \smile_{1} x_{3}+x_{1} \smile_{1} x_{3} \\
& -P\left(\partial\left(x_{3} \smile_{1} x_{1}\right) \cdot\left[\tau_{n_{1}, n_{3}}\right] \mid x_{2} \cdot x_{3}\right)_{H} \\
& \text { M3 }=x_{1} \smile_{1} x_{3}+x_{2} \smile_{1} x_{3} \\
& -P(\partial\left(x_{1} \smile_{1} x_{3}\right) \mid \underbrace{\partial\left(x_{2} \smile_{1} x_{3}\right)}_{\text {E2 })})_{H} \\
& -P\left(\partial\left(\left(x_{3} \smile_{1} x_{1}\right) \cdot\left[\tau_{n_{1}, n_{3}}\right]\right) \mid x_{2} \cdot x_{3}\right)_{H} \\
& \text { E3 }=x_{1} \smile_{1} x_{3}+x_{2} \smile_{1} x_{3} \\
& +P\left(\partial\left(x_{1} \smile_{1} x_{3}\right) \mid x_{3} \cdot x_{2} \cdot\left[\tau_{n_{2}, n_{3}}\right]\right)_{H} .
\end{aligned}
$$

Equation (5) follows from

$$
\begin{aligned}
& x_{1} \smile_{1}\left(x_{2} \cdot x_{3}\right) \stackrel{\text { E3 }}{=}-\left(\left(x_{2} \cdot x_{3}\right) \smile_{1} x_{1}\right) \cdot\left[\tau_{n_{1}, n_{2}+n_{3}}\right] \\
& \underline{E \text { E5/A2/S1 }}=-\left(x_{2} \cdot\left(x_{3} \smile_{1} x_{1}\right)\right) \cdot\left[\tau_{n_{1}, n_{2}+n_{3}}\right] \\
& -\left(\left(x_{2} \smile_{1} x_{1}\right) \cdot x_{3}\right) \cdot\left[1_{n_{2}} \times \tau_{n_{3}, n_{1}}\right] \cdot\left[\tau_{n_{1}, n_{2}+n_{3}}\right] \\
& \text { E[1E1|A1|A2|S1] }=\left(x_{2} \cdot\left(x_{1} \smile_{1} x_{3}\right)\right) \cdot\left[1_{n_{2}} \times \tau_{n_{3}, n_{1}}\right] \cdot\left[\tau_{n_{1}, n_{2}+n_{3}}\right] \\
& +\left(\left(x_{1} \smile_{1} x_{2}\right) \cdot x_{3}\right) \cdot\left[\tau_{n_{2}, n_{1}} \times 1_{n_{3}}\right] \cdot\left[1_{n_{2}} \times \tau_{n_{3}, n_{1}}\right] \cdot\left[\tau_{n_{1}, n_{2}+n_{3}}\right] \\
& \underline{\text { S33 }}=\left(x_{2} \cdot\left(x_{1} \smile_{1} x_{3}\right)\right) \cdot\left[\tau_{n_{1}, n_{2}} \times 1_{n_{3}}\right]+\left(x_{1} \smile_{1} x_{2}\right) \cdot x_{3} .
\end{aligned}
$$


Equation (6) follows from

$$
\begin{aligned}
&\left(\left(x_{3} \cdot x_{1}\right) \smile_{1} x_{2}\right) \cdot\left[\tau_{n_{1}+n_{2}, n_{3}}\right]+\left(x_{1} \cdot x_{2}\right) \smile_{1} x_{3} \\
&=\left(\left(x_{3} \smile_{1} x_{2}\right) \cdot x_{1}\right) \cdot\left[1_{n_{3}} \times \tau_{n_{1}, n_{2}}\right] \cdot\left[\tau_{n_{1}+n_{2}, n_{3}}\right]+\left(x_{3} \cdot\left(x_{1} \smile_{1} x_{2}\right)\right) \cdot\left[\tau_{n_{1}+n_{2}, n_{3}}\right] \\
&+\left(\left(x_{1} \smile_{1} x_{3}\right) \cdot x_{2}\right) \cdot\left[1_{n_{1}} \times \tau_{n_{2}, n_{3}}\right]+x_{1} \cdot\left(x_{2} \smile_{1} x_{3}\right) \\
& \underline{\text { E5 } 6.55)=}-\left(\left(x_{2} \smile_{1} x_{3}\right) \cdot x_{1}\right) \cdot\left[\tau_{n_{1}, n_{2}+n_{3}}\right] \\
&+\left(x_{1} \smile_{1}\left(x_{3} \cdot x_{2}\right)\right) \cdot\left[1_{n_{1}} \times \tau_{n_{2}, n_{3}}\right]+x_{1} \cdot\left(x_{2} \smile_{1} x_{3}\right) \\
&=\left(x_{1} \smile_{1}\left(x_{3} \cdot x_{2}\right)\right) \cdot\left[1_{n_{1}} \times \tau_{n_{2}, n_{3}}\right]+x_{1} \smile_{1} \partial\left(x_{2} \smile_{1} x_{3}\right) \\
&+P\left(\partial\left(x_{1} \smile_{1}\left(x_{3} \cdot x_{2}\right)\right) \cdot\left[1_{n_{1}} \times \tau_{n_{2}, n_{3}}\right] \partial\left(\left(x_{2} \smile_{1} x_{3}\right) \cdot x_{1}\right) \cdot\left[\tau_{n_{1}, n_{2}+n_{3}}\right]\right)_{H} \\
& \text { M3 } x_{1} \smile_{1}\left(x_{2} \cdot x_{3}\right)
\end{aligned}
$$

We are now ready to prove Theorem 6.4.

Proof of Theorem 6.4. We assume without loss of generality that all representatives chosen in $B_{*, 0}$ are in Ker $H$.

(T7) By (E2, A3) we can take

$$
\begin{aligned}
& \overline{a b}=\overline{b a} \cdot\left[\tau_{|a|,|b|}\right]+\bar{a} \smile_{1} \bar{b} \\
& \overline{b c}=\overline{c b} \cdot\left[\tau_{|b|,|c|}\right]+\bar{b} \smile_{1} \bar{c},
\end{aligned}
$$

and so we do in this proof, therefore

$$
\begin{aligned}
& \text { (a) }-\overline{a b} \cdot \bar{c}+\bar{a} \cdot \overline{b c} \stackrel{\text { E2 } 2 \text { 6.5 } 3)}{=}-(\bar{a} \cdot \bar{b}) \smile_{1} \bar{c}-(\bar{c} \cdot \overline{a b}) \cdot\left[\tau_{|a|+|b|,|c|}\right] \\
& +(\overline{b c} \cdot \bar{a}) \cdot\left[\tau_{|a|,|b|+|c|}\right]+\bar{a} \smile_{1}(\bar{b} \cdot \bar{c}) \\
& =\quad-(\bar{a} \cdot \bar{b}) \smile_{1} \bar{c}-\left(\bar{c} \cdot\left(\overline{b a} \cdot\left[\tau_{|a|,|b|}\right]+\bar{a} \smile_{1} \bar{b}\right)\right) \cdot\left[\tau_{|a|+|b|,|c|}\right] \\
& +\left(\left(\overline{c b} \cdot\left[\tau_{|b|,|c|}\right]+\bar{b} \smile_{1} \bar{c}\right) \cdot \bar{a}\right) \cdot\left[\tau_{|a|,|b|+|c|}\right]+\bar{a} \smile_{1}(\bar{b} \cdot \bar{c}) \\
& \left(\underline{\text { A1A22E16.5 } 1)}=-(\bar{a} \cdot \bar{b}) \smile_{1} \bar{c}-\left(\bar{c} \cdot\left(\bar{a} \smile_{1} \bar{b}\right)\right) \cdot\left[\tau_{|a|+|b|,|c|}\right]\right. \\
& +(-\bar{c} \cdot \overline{b a}+\overline{c b} \cdot \bar{a}) \cdot\left[\tau_{|b|,|c|} \times 1_{|a|}\right] \cdot\left[\tau_{|a|,|b|+|c|}\right] \\
& +\left(\left(\bar{b} \smile_{1} \bar{c}\right) \cdot \bar{a}\right) \cdot\left[\tau_{|a|,|b|+|c|}\right]+\bar{a} \smile_{1}(\bar{b} \cdot \bar{c})
\end{aligned}
$$

The element $-\bar{c} \cdot \overline{b a}+\overline{c b} \cdot \bar{a}$ represents $-\langle c, b, a\rangle$, so it is in Ker $\partial$, in particular by Lemma 5.4

$(-\bar{c} \cdot \overline{b a}+\overline{c b} \cdot \bar{a}) \cdot\left[\tau_{|b|,|c|} \times 1_{|a|}\right] \cdot\left[\tau_{|a|,|b|+|c|}\right]=(-1)^{|a||b|+|b||c|+|c||a|+1}(-\overline{c b} \cdot \bar{a}+\bar{c} \cdot \overline{b a})$.

Since Ker $\partial$ is central we only need to see that the rest of factors in the previous equation cancel, and this follows from (6.5.6) since

$$
\begin{aligned}
& -\left(\bar{c} \cdot\left(\bar{a} \smile_{1} \bar{b}\right)\right) \cdot\left[\tau_{|a|+|b|,|c|}\right]+\left(\left(\bar{b} \smile_{1} \bar{c}\right) \cdot \bar{a}\right) \cdot\left[\tau_{|a|,|b|+|c|}\right] \\
& \qquad \begin{array}{l}
\text { E3 } \\
\text { (E5) }
\end{array}=-\left(\bar{c} \cdot\left(\bar{a} \smile_{1} \bar{b}\right)\right) \cdot\left[\tau_{|a|+|b|,|c|}\right]-\left(\left(\bar{c} \smile_{1} \bar{b}\right) \cdot \bar{a}\right) \cdot\left[\tau_{|b|,|c|} \times 1_{|a|}\right] \cdot\left[\tau_{|a|,|b|+|c|}\right] \\
& \left.=-(\bar{c} \cdot \bar{a}) \smile_{1} \bar{b}\right) \cdot\left[\tau_{|a|+|b|,|c|}\right] .
\end{aligned}
$$

(T8) The following equation is obtained from the first equality in (a) above by inserting in the middle two elements which cancel

$$
\begin{aligned}
-\overline{a b} \cdot \bar{c}+\bar{a} \cdot \overline{b c}= & -(\bar{a} \cdot \bar{b}) \smile_{1} \bar{c}-(\bar{c} \cdot \overline{a b}) \cdot\left[\tau_{|a|+|b|,|c|}\right]+(\overline{c a} \cdot \bar{b}) \cdot\left[\tau_{|a|+|b|,|c|}\right] \\
& -\underbrace{(\bar{c}) \bar{b}}_{\left(\overline{\mathrm{E} 2}=(\bar{c} \cdot \overline{c a}) \cdot\left[\tau_{|c|+|a|,|b|}\right]+(\bar{c} \cdot \bar{a}) \smile_{1} \bar{b}\right.} \cdot\left[\tau_{|a|+|b|,|c|}\right]+(\overline{b c} \cdot \bar{a}) \cdot\left[\tau_{|a|,|b|+|c|}\right]+\bar{a} \smile_{1}(\bar{b} \cdot \bar{c}) .
\end{aligned}
$$

By Lemma 5.4

$$
\begin{aligned}
& (-\bar{c} \cdot \overline{a b}+\overline{c a} \cdot \bar{b}) \cdot\left[\tau_{|a|+|b|,|c|}\right] \in-(-1)^{|a||c|+|b||c|}\langle c, a, b\rangle, \\
& (-\bar{b} \cdot \overline{c a}+\overline{b c} \cdot \bar{a}) \cdot\left[\tau_{|a|,|b|+|c|}\right] \in-(-1)^{|a||b|+|a||c|}\langle b, c, a\rangle,
\end{aligned}
$$

therefore, since Ker $\partial$ is central, (T8) follows from (6.5.6). 
Let us now check simultaneously (T9) and (T10). By (E2, A3) we can take

$$
\overline{(2 a)}=\bar{a}+\bar{a} .
$$

Moreover, by (S4|E2|A3|A1) for $|a|$ odd we can take

$$
\begin{aligned}
& \overline{a(2 a)}=\overline{(2 a) a}=-\bar{a}^{2} \cdot\left[\hat{\tau}_{|a|,|a|}\right]+\bar{a} \smile_{1} \bar{a} . \\
& -\overline{a b} \cdot \bar{a}+\bar{a} \cdot \overline{b a}=-\left(\bar{a} \smile_{1} \bar{b}\right) \cdot \bar{a}-(\overline{b a} \cdot \bar{a}) \cdot\left[\tau_{|a|,|b|} \times 1_{|a|}\right] \\
& \text { A1[E1|6.5 } 3) \quad+(\overline{b a} \cdot \bar{a}) \cdot \underbrace{\left[\tau_{|a|,|b|+|a|}\right]}+\bar{a} \smile_{1}(\bar{b} \cdot \bar{a}) \\
& \text { [S3] }=\left[1_{|b|} \times \tau_{|a|,|a|}\right] \cdot\left[\tau_{|a|,|b|} \times 1_{|a|}\right] \\
& \left.\underline{\underline{\mathrm{A} 1 \mid \mathrm{S} 4}}=-\left(\bar{a} \smile_{1} \bar{b}\right) \cdot \bar{a}-(\mathrm{c})\right\}=\left\{\begin{array}{l}
(\overline{b a} \cdot(\bar{a}+\bar{a})) \cdot\left[\tau_{|a|,|b|} \times 1_{|a|}\right],|a| \text { odd, } \\
0,|a| \text { even. }
\end{array}\right. \\
& -(\overline{b a} \cdot \bar{a}) \cdot \partial\left[S^{|b|} \wedge \hat{\tau}_{|a|,|a|}\right] \cdot\left[\tau_{|a|,|b|} \times 1_{|a|}\right]+\bar{a} \smile_{1}(\bar{b} \cdot \bar{a}) \\
& \underline{\text { A3 [E1|E5 }}=-\left(\bar{a} \smile_{1} \bar{b}\right) \cdot \bar{a}-(\mathrm{c})-\left(\bar{b} \cdot\left(\bar{a}^{2} \cdot\left[\hat{\tau}_{|a|,|a|}\right]\right)\right) \cdot\left[\tau_{|a|,|b|} \times 1_{|a|}\right] \\
& +\left(\bar{b} \cdot\left(\bar{a} \smile_{1} \bar{a}\right)\right) \cdot\left[\tau_{|a|,|b|} \times 1_{|a|}\right]+\left(\bar{a} \smile_{1} \bar{b}\right) \cdot \bar{a} \\
& \underline{\mathrm{A} 1|\mathrm{~A} 2| \mathrm{S} 10}=-\left(\bar{a} \smile_{1} \bar{b}\right) \cdot \bar{a}-(\mathrm{c}) \\
& +\left(\bar{b} \cdot\left(-\bar{a}^{2} \cdot\left[\hat{\tau}_{|a|,|a|}\right]+\bar{a} \smile_{1} \bar{a}\right)\right) \cdot\left[\tau_{|a|,|b|} \times 1_{|a|}\right]+\left(\bar{a} \smile_{1} \bar{b}\right) \cdot \bar{a}
\end{aligned}
$$

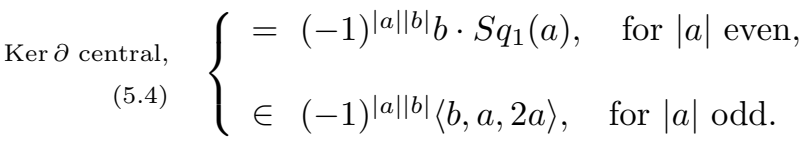

In order to check (T11) let $a, b \in h_{0} B_{2 n, *}, \tau=\tau_{2 n, 2 n}$, and $\hat{\tau}=\hat{\tau}_{2 n, 2 n}$. Using the "bilinearity mod $P$ " of the product and the cup-one product we obtain

$$
\begin{gathered}
-(\bar{a}+\bar{b})^{2} \cdot[\hat{\tau}]+(\bar{a}+\bar{b}) \smile_{1}(\bar{a}+\bar{b}) \\
=-\bar{b}^{2} \cdot[\hat{\tau}]-(\bar{a} \cdot \bar{b}) \cdot[\hat{\tau}]-(\bar{b} \cdot \bar{a}) \cdot[\hat{\tau}]-\bar{a}^{2} \cdot[\hat{\tau}]-(\mathrm{b}) \\
+\bar{a} \smile_{1} \bar{a}+\bar{b} \smile_{1} \bar{a}+\bar{a} \smile_{1} \bar{b}+\bar{b} \smile_{1} \bar{b}+(\mathrm{c}) .
\end{gathered}
$$

The central elements (b) and (c) are

$$
\begin{aligned}
(\mathrm{b})= & P\left(\left(\bar{b} \cdot \bar{a}+\bar{a} \cdot \bar{b}+\bar{b}^{2} \mid \bar{a}^{2}\right)_{H} \cdot H \partial[\hat{\tau}]\right)+P\left(\left(\bar{a} \cdot \bar{b}+\bar{b}^{2} \mid \bar{b} \cdot \bar{a}\right)_{H} \cdot H \partial[\hat{\tau}]\right) \\
& +P\left(\left(\bar{a} \cdot \bar{b}+\bar{b}^{2} \mid \bar{a} \cdot \bar{b}\right)_{H} \cdot H \partial[\hat{\tau}]\right), \\
(\mathrm{c})= & P\left(\partial\left(\bar{a} \smile_{1} \bar{a}\right) \mid(\bar{a} \cdot \bar{b}) \cdot[\tau]\right)_{H}+P\left(\partial\left(\bar{a} \smile_{1} \bar{b}\right) \mid \bar{b}^{2} \cdot[\tau]\right)_{H} \\
& +P\left(\partial\left((\bar{a}+\bar{b}) \smile_{1} \bar{a}\right) \mid(\bar{b} \cdot(\bar{a}+\bar{b})) \cdot[\tau]\right)_{H} .
\end{aligned}
$$

In the middle of equation (a) we find the formula for $S q_{1}(a)$ which is central in $B_{4 n, 1}$, so we can move it to the end of the equation, as (b) and (c). Moreover, by [BM06c, Lemma 7.4] and (S5IS6) we have

$$
n P(1 \mid 1)_{H}=[\tau] \cdot[\hat{\tau}]+[\hat{\tau}]
$$


This formula is used in the following equation.

$$
\begin{aligned}
& \bar{b} \smile_{1} \bar{a}+\bar{a} \smile_{1} \bar{b} \stackrel{\stackrel{\mathrm{E} 3}{=}}{=}-\left(\bar{a} \smile_{1} \bar{b}\right) \cdot[\tau]+\bar{a} \smile_{1} \bar{b} \\
& \text { A1 }=\left(\bar{a} \smile_{1} \bar{b}\right) \cdot(-[\tau]+1) \\
& \text { [S4] }=\left(\bar{a} \smile_{1} \bar{b}\right) \cdot \partial[\hat{\tau}] \\
& \overline{\mathrm{A} 3 \mathrm{E} 2 \mathrm{2}}=(-(\bar{b} \cdot \bar{a}) \cdot[\tau]+\bar{a} \cdot \bar{b}) \cdot[\hat{\tau}] \\
& \overline{\mathrm{A} 2}=-(\bar{b} \cdot \bar{a}) \cdot([\tau] \cdot[\hat{\tau}])+(\bar{a} \cdot \bar{b}) \cdot[\hat{\tau}] \\
& +\underbrace{P\left((-\bar{a} \cdot \bar{b}+(\bar{b} \cdot \bar{a}) \cdot[\tau] \mid(\bar{b} \cdot \bar{a}) \cdot[\tau])_{H} \cdot H \partial[\hat{\tau}]\right)}_{=(\mathrm{e})} \\
& (\mathrm{d} \widehat{\mathrm{A} 4 \mid \mathrm{A} 8}=(\bar{b} \cdot \bar{a}) \cdot[\hat{\tau}]+(\bar{a} \cdot \bar{b}) \cdot[\hat{\tau}]+(\mathrm{e})+n \underbrace{P(\bar{b} \cdot \bar{a} \mid \bar{b} \cdot \bar{a})_{H}}_{=a \cdot b \cdot \eta} .
\end{aligned}
$$

This shows that (a) simplifies to give the following equation

$$
S q_{1}(a+b)=S q_{1}(a)+S q_{1}(b)+n \cdot a \cdot b \cdot \eta+(\mathrm{b})+(\mathrm{c})+(\mathrm{e}) .
$$

Now one uses $\mathrm{A} 8|\mathrm{S3}| 5.1$ and the elementary properties of quadratic pair modules to check that

$$
\text { (b) }+(\mathrm{c})+(\mathrm{e})=P(\bar{b} \cdot \bar{a} \mid \bar{b} \cdot \bar{a})_{H}=a \cdot b \cdot \eta \text {, }
$$

hence we are done.

Finally (T12) will follow from (5.9]6.2) once we check that for the cup-one square $\overline{S q}_{1}(a \cdot b)$ associated to the following lift of $\tau_{|a|+|b|,|a|+|b|}$

$$
\tilde{\tau}=\left(S^{|a|} \wedge \hat{\tau}_{|b|,|a|}^{-1} \wedge S^{|b|}\right)\left(S^{2|a|} \wedge \hat{\tau}_{|b|,|b|}\right)\left(\hat{\tau}_{|a|,|a|} \wedge S^{2|b|}\right)\left(S^{|a|} \wedge \hat{\tau}_{|b|,|a|} \wedge S^{|b|}\right)
$$

the following formula holds.

$$
\overline{S q}_{1}(a \cdot b)=a^{2} \cdot S q_{1}(b)+S q_{1}(a) \cdot b^{2} .
$$

The following equation holds.

$$
\begin{aligned}
& {[\tilde{\tau}] \stackrel{\text { S5 }}{=}\left[1_{|a|} \times \tau_{|a|,|b|} \times 1_{|b|}\right] \cdot\left[\left(S^{2|a|} \wedge \hat{\tau}_{|b|,|b|}\right)\left(\hat{\tau}_{|a|,|a|} \wedge S^{2|b|}\right)\left(S^{|a|} \wedge \hat{\tau}_{|b|,|a|} \wedge S^{|b|}\right)\right]} \\
& +\left[S^{|a|} \wedge \hat{\tau}_{|b|,|a|}^{-1} \wedge S^{|b|}\right] \\
& \underline{5.3 \mid \mathrm{A} 1]}=\left[1_{|a|} \times \tau_{|a|,|b|} \times 1_{|b|}\right] \cdot\left[\left(S^{2|b|} \wedge \hat{\tau}_{|b|,|b|}\right)\left(\hat{\tau}_{|a|,|a|} \wedge S^{2|b|}\right)\right] \cdot\left[1_{|a|} \times \tau_{|b|,|a|} \times 1_{|b|}\right] \\
& +\underbrace{\left[1_{|a|} \times \tau_{|a|,|b|} \times 1_{|b|}\right] \cdot\left[S^{|a|} \wedge \hat{\tau}_{|b|,|a|} \wedge S^{|b|}\right]+\left[S^{|a|} \wedge \hat{\tau}_{|b|,|a|}^{-1} \wedge S^{|b|}\right]}_{[5.2[S 5 \mid} \\
& \text { (S5) }=\left[1_{|a|} \times \tau_{|a|,|b|} \times 1_{|b|}\right] \cdot\left(\left[1_{2|a|} \times \tau_{|b|,|b|}\right] \cdot\left[\hat{\tau}_{|a|,|a|} \wedge S^{2|b|}\right]\right. \\
& \left.+\left[S^{2|a|} \wedge \hat{\tau}_{|b|,|b|}\right]\right) \cdot\left[1_{|a|} \times \tau_{|b|,|a|} \times 1_{|b|}\right] .
\end{aligned}
$$

This equation is used below. We will also use the notation

$$
\begin{aligned}
\left({ }^{*}\right) & =P\left(\left(\bar{a} \cdot \partial\left(\bar{b} \smile_{1} \bar{a}\right) \cdot \bar{b} \mid\left(\bar{a}^{2} \cdot \bar{b}^{2}\right) \cdot\left[1_{|a|} \times \tau_{|b|,|a|} \times 1_{|b|}\right]\right)_{H} \cdot H \partial[\tilde{\tau}]\right) \\
\underline{\mathrm{M} 3[5.1 \mid \mathrm{A} 8} & =\left[\left(\bar{a} \cdot\left(\bar{b} \smile_{1} \bar{a}\right) \cdot \bar{b}\right) \cdot\left[\tau_{|a|+|b|,|a|+|b|}\right],-\left(\bar{a}^{2} \cdot \bar{b}^{2}\right) \cdot\left[1_{|a|} \times \tau_{|b|,|a|} \times 1_{|b|}\right] \cdot[\tilde{\tau}]\right] .
\end{aligned}
$$


Now the formula follows from the following equations.

$$
\begin{aligned}
& -(\bar{a} \cdot \bar{b})^{2} \cdot[\tilde{\tau}]+(\bar{a} \cdot \bar{b}) \smile_{1}(\bar{a} \cdot \bar{b}) \\
& \left(\begin{array}{c}
\text { E2|A1/A2[E1 } \\
\text { E5[6.54 } 4 \text { A6]E6 }
\end{array}\right)=-\left(\left(\bar{a}^{2} \cdot \bar{b}^{2}\right) \cdot\left[1_{|a|} \times \tau_{|b|,|a|} \times 1_{|b|}\right]+\bar{a} \cdot \partial\left(\bar{b} \smile_{1} \bar{a}\right) \cdot \bar{b}\right) \cdot[\tilde{\tau}]
\end{aligned}
$$

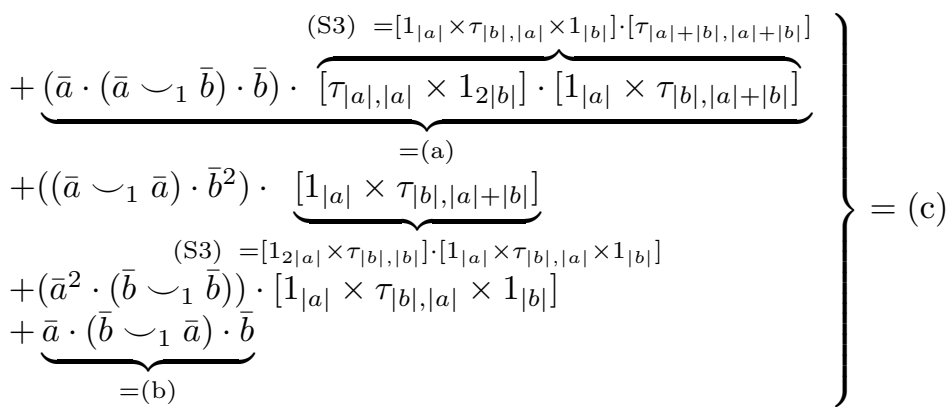

$$
\begin{aligned}
& \text { A25.11 } \\
& =-\underbrace{\left(\bar{a} \cdot \partial\left(\bar{b} \smile_{1} \bar{a}\right) \cdot \bar{b}\right) \cdot[\tilde{\tau}]}-\left(\bar{a}^{2} \cdot \bar{b}^{2}\right) \cdot\left[1_{|a|} \times \tau_{|b|,|a|} \times 1_{|b|}\right] \cdot[\tilde{\tau}] \\
& \text { A33 }=\left(\bar{a} \cdot\left(\bar{b} \smile_{1} \bar{a}\right) \cdot \bar{b}\right) \cdot \partial[\tilde{\tau}] \\
& \underline{\mathrm{S} 4 \mid \mathrm{A} 1]}=-\underbrace{\left.\left(\bar{b} \smile_{1} \bar{a}\right) \cdot \bar{b}\right) \cdot\left[\tau_{|a|+|b|,|a|+|b|]}\right.}_{\text {cancels with }(\mathrm{a}) \text { and }\left({ }^{*}\right) \text { by }[\mathrm{E} 1 \mathrm{E} \cdot \mathrm{E} 3|\mathrm{~A} 1| \mathrm{A} 2} \\
& +\underbrace{\left(\bar{a} \cdot\left(\bar{b} \smile_{1} \bar{a}\right) \cdot \bar{b}\right)}_{\text {cancels with (b) }} \\
& -(*)+(\mathrm{c}) \\
& \text { E1[6.5 } 2 \text { A1 } 1 \mathrm{A2} 2=\left(-\bar{a}^{2} \cdot\left(\bar{b}^{2} \cdot\left[\hat{\tau}_{|b|,|b|}\right]\right)-\left(\bar{a}^{2} \cdot\left[\hat{\tau}_{|a|,|a|}\right]\right) \cdot\left(\bar{b}^{2} \cdot\left[\tau_{|b|,|b|}\right]\right)\right.
\end{aligned}
$$

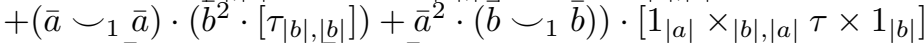

$$
\begin{aligned}
& \text { A6 A1 A2 } \\
& =\left(\bar{a}^{2} \cdot\left(-\bar{b}^{2} \cdot\left[\hat{\tau}_{|b|,|b|}\right]+\bar{b} \smile_{1} \bar{b}\right)\right. \\
& \left.+\left(-\bar{a}^{2} \cdot\left[\hat{\tau}_{|a|,|a|}\right]+\bar{a} \smile_{1} \bar{a}\right) \cdot\left(\bar{b}^{2} \cdot\left[\tau_{|b|,|b|}\right]\right)\right) \cdot\left[1_{|a|} \times \tau_{|b|,|a|} \times 1_{|b|}\right] \\
& =a^{2} \cdot S q_{1}(b)+S q_{1}(a) \cdot b^{2} \text {. }
\end{aligned}
$$

\section{REFERENCES}

[Ale72] J. C. Alexander, Cobordism Massey products, Trans. Amer. Math. Soc. 166 (1972), 197-214.

[BJP05] H.-J. Baues, M. Jibladze, and T. Pirashvili, Quadratic algebra of square groups, Preprint of the Max-Planck-Institut für Mathematik MPIM2006-9, http://arxiv.org/abs/math.CT/0601777, 2005.

[BJP06] , Third Mac Lane cohomology, Preprint of the Max-Planck-Institut für Mathematik MPIM2006-50, http://arxiv.org/abs/math.KT/0604447, 2006.

[BM06a] H.-J. Baues and F. Muro, Secondary algebras associated to ring spectra, Preprint of the Max-Planck-Institut für Mathematik MPIM2006-137, http://arxiv.org/abs/math.AT/0610523, 2006.

[BM06b] Smash products for secondary homotopy groups, Preprint of the Max-PlanckInstitut für Mathematik MPIM2006-38, http://arxiv.org/abs/math.AT/0604031, 2006.

[BM06c] The symmetric action on secondary homotopy groups, Preprint of the Max-Planck-Institut für Mathematik MPIM2006-37, http://arxiv.org/abs/math.AT/0604030, 2006.

[BMMS86] R. R. Bruner, J. P. May, J. E. McClure, and M. Steinberger, $H_{\infty}$ ring spectra and their applications, Lecture Notes in Mathematics, vol. 1176, Springer-Verlag, Berlin, 1986.

[BP99] H.-J. Baues and T. Pirashvili, Quadratic endofunctors of the category of groups, Advances in Math. 141 (1999), 167-206.

[Hel68] A. Heller, Stable homotopy categories, Bull. Amer. Math. Soc. 74 (1968), 28-63.

[MMSS01] M. A. Mandell, J. P. May, S. Schwede, and B. Shipley, Model categories of diagram spectra, Proc. London Math. Soc. (3) 82 (2001), no. 2, 441-512.

[Sag06] S. Sagave, Universal toda brackets of ring spectra, Ph.D. thesis, University of Bonn, 2006 . 
[Sch04] S. Schwede, Morita theory in abelian, derived and stable model categories, Structured ring spectra, London Math. Soc. Lecture Note Ser., vol. 315, Cambridge Univ. Press, Cambridge, 2004, pp. 33-86.

[Tod62] H. Toda, Composition methods in homotopy groups of spheres, Annals of Mathematics Studies, No. 49, Princeton University Press, Princeton, N.J., 1962.

Max-Planck-Institut für Mathematik, Vivatsgasse 7, 53111 Bonn, Germany

E-mail address: baues@mpim-bonn.mpg.de, muro@mpim-bonn.mpg.de 